\title{
Fusion Policy Advisory Committee (FPAC)
}

\section{FINAL REPORT}

September 1990

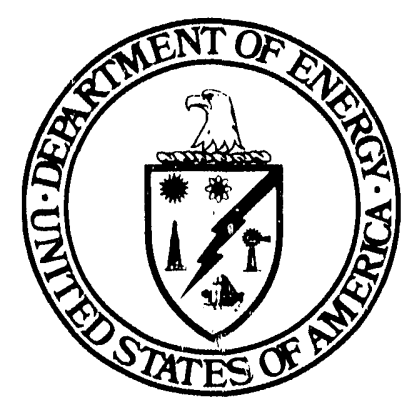

Report of the Technical Panel on

Magnetic Fusion of the Energy Research

Advisory Board

Washington, D.C. 20585 


\title{
Fusion Policy Advisory Committee
}

\author{
United States Department of Energy \\ 1000 Independence Avenue, S.W. \\ Washington, D.C. 20585
}

(202) 586-5444

September 25, 1990

\begin{abstract}
Admiral James D. Watkins
The Secretary of Energy

U.S. Department of Energy

Washington, D.C. 20585
\end{abstract}

\section{Dear Admiral Watkins:}

I am pleased to submit to you the final report of your Fusion Policy Advisory Committee. It presents a fusion policy that the Committee believes is in the best interests of the Nation in its pursuit of a secure energy future. After subjecting the fusion program to close scrutiny, the Committee believes that there are persuasive reasons for the U.S. to commit to a responsible, goaloriented fusion energy development program. The successful development of this energy source would be of great value to the U.S. and indeed to the citizens of all Nations.

This report presents a conceptual program plan that can achieve the goals of at least one operating Demonstration Power Plant by 2025 and at least one operating Commercial Power Plant by 2040 . This plan is fully supported by the Committee. The Committee's confidence in the future of fusion energy stems from the impressive progress that has been made in fusion energy research worldwide. We believe that the U.S. fusion program is technically ready in this decade to construct devices to demonstrate significant fusion power production in a burning tokamak plasma and ignition in an inertially confined pellet.

In view of the tight funding climate and the requirement in the conceptual plan for an immediate ramp-up of funding, you requested next step program options which would have lower immediate effects on the fusion budget. These are included in the report, together with the rationale for selecting certain reduced next step development paths. However, the first funding increments for new facilities in the constrained program options are essential for fusion to be an energy program. If these incremerits are not forthcoming, the program would remain only a research effort without reasonably-timed energy objectives.

The Committee believes that fusion has very favorable environmental, safety and fuel-availability potentialities. It holds the promise of an acceptable, limitless energy source. Fusion's urgency will be further increased if fossil fuel and nuclear fission become environmentally unacceptable or if the price of these fuels rises dramatically.

\section{STRATEGY AND RECOMMENDATIONS}

1. The fusion energy program should have two distinct and separate approaches, magnetic fusion energy (MFE) and inertial fusion energy (IFE), both aimed at the same goal of fusion energy production. Both should plan for major facilities along the lines of the Committee's conceptual plan in the report. 
2. Both MFE and IFE should participate in international collaborations. Indeed the U.S. should work to strengthen international collaboration well beyond the current activity. For MFE, a unique opportunity exists to broaden international collaboration at this time. This will require unprecedented investment in shared facilities, some of which will be located overseas. The U.S. should face this issue squarely and take the lead in negotiating a world effort while insuring a strong domestic program.

3. The U.S. should take an even-handed approach in strengthening its national and international efforts in MFE by participating as an equal partner in the ITER Engineering Design Activity (EDA) and by authorizing the construction of the Burning Plasma Experiment in the FY 1992 budget. Strong reliance by the U.S. on an international MFE program should be balanced by a strengthened program at home, comparable to that of our major partners. A weak U.S. program, now substantially below the funding level of Europe, will limit American influence on the ITER project and compromise the ability of the U.S. fusion program to capitalize on what is learned from ITER. The U.S. should propose to locate the ITER EDA in this country, while accepting the possibility that it could be located elsewhere.

4. The first priority for the inertial fusion program (both Energy and Defense) is the resolution of the target physics issues, to be accomplished by an augmented 2 to 3 year program on Precision Nova. The proposed Nova Upgrade should be authorized in FY 1992. Then, if the milestones are met with Precision Nova, the Nova Upgrade construction can begin in FY 1994 to demonstrate ignition and moderate gain before the year 2000 . To provide further data in evaluating direct drive prospects we endorse construction of the Omega Upgrade at the University of Rochester. We strongly urge augmenting the program for developing a driver of suitable efficiency and reliabilitiy to be useful for energy production, with emphasis un heavy ions. The IFE program should take maximum advantage of the ICF research programs conducted for defense.

5. Both MFE and IFE should increase indusirial participation to permit an orderly transition to an energy program witi strong emphasis on technology development. Both require sirong university programs to ensure the education of fusion scientists and engineers, to advance fundamental understanding, and to develop fusion technologies and new concepts:

All of the members of the Fusion Policy Advisory Committee wish to thank you and many members of your staff, staff members of the National Laboratories and other DOE contractors, and many other interested parties for the time and help they have given. Also, I wish to thank all of the members of the Committee for their dedicated work.

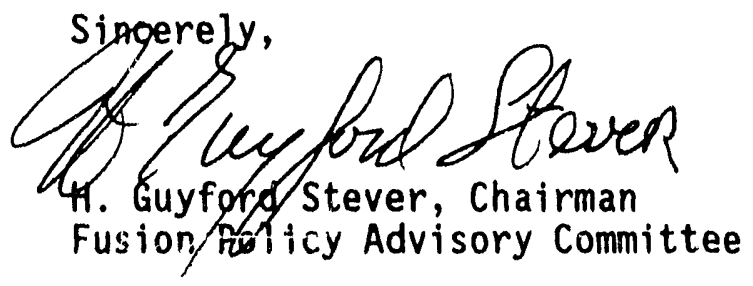




\section{FUSION POLICY ADVISORY COMMITTEE MEMBERS}

Dr. H. Guyford Stever, Chairman

Foreign Secretary (Retired)

National Academy of Engineering

Dr. Roger Batzel

Director (Retired)

Lawrence Livermore National Laboratory

Dr. Ira B. Bernstein

Professor of Applied Physics

Yale University

Dr. Robert W. Conn

Professor of Nuclear Engineering

Institute of Plasma and Fusion Research

University of California, Los Angeles

Dr. E. Linn Draper, Jr.

Chairman of the Board and President

Gulf States Utilities

Dr. Harold K. Forsen

Senior Vice President and Manager of R\&D

Bechtel Group

Dr. John S. Foster, Jr.

Vice President for Science and Technology

(Retired)

TRW

Dr. T. Kenneth Fowler

Chai:man

Depirtment of Nuclear Engineering

University of California, Berkeley

Dr. Melvin B. Gottlieb

Director (Retired)

Princeton Plasma Physics Laboratory

Dr. William Herrmannsfeldt

Stanford Linear Accelerator Center

Dr. Charles F. Kennel

Professor of Physics

University of California, Los Angeles

Dr. Arthur K. Kerman

Director, Laboratory for Nuclear Science

Massachusetts Institute of Technology
Dr. Kenneth L. Kliewer

Dean, School of Sciences

Purdue University

Mr. John W. Landis

Senior Vice President and Director

Stone and Webster Engineering

Corporation

Dr. R. Bruce Miller

TITAN Corporation

Dr. Barrett H. Ripin

Head, Space Plasma Branch

Naval Research Laboratory

Dr. Marshall N. Rosenbluth

Professor of Physics

University of California, San Diego

Dr. Robert Sproull

President Emeritus

University of Rochester

Dr. Richard Wilson

Mallinckrodt Professor of Physics

Lyman Laboratory of Physics

Harvard University

\section{TECHINICAL STAFF}

Executive Director

Dr. James Kane

University of California

Dr. Thomas Finn

U.S. Department of Energy

Dr. Loren Schmid

Paci : Northwest Laboratory

Mr. Robert Jones

U.S. Department of Energy

Mr. James Yeck

U.S. Department of Energy

Princeton Area Office 
CHAPTER I CONCLUSIONS AND RECOMMENDATIONS $\ldots \ldots \ldots \ldots \ldots \ldots \ldots \ldots \ldots$

A. INTRODUCTION................................... 1

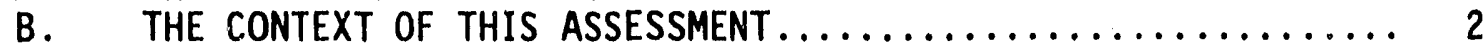

C. POLICY.......................................... 3

D. STRATEGY........................................ 4

E. PROGRAM AND BUDGET RECOMMENUATIONS $\ldots \ldots \ldots \ldots \ldots \ldots \ldots \ldots, 5$

E.1 Magnetic Fusion Energy Development............... 5

E.2 Inertial Fusion Energy Development............... 5

F. ORGANIZATION.................................... 6

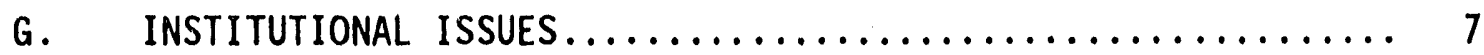

G.1 Industrial Participation......................., 7

G.2 University Participation....................... 7

G.3 Laboratory Participation...................... 7

H. MANAGEMENT ISSUES $\ldots \ldots \ldots \ldots \ldots \ldots \ldots \ldots \ldots \ldots \ldots \ldots, 8$

H.1 Program Management........................ 8

H.2 Inertial Fusion Energy Program Coordiriation.......... 8

H.3 Classification and Technology Transfer............. 8

CHAPTER II COMMON ISSUES $\ldots \ldots \ldots \ldots \ldots \ldots \ldots \ldots \ldots \ldots \ldots \ldots \ldots \ldots \ldots \ldots$

A. INTRODUCTION.................................. 11

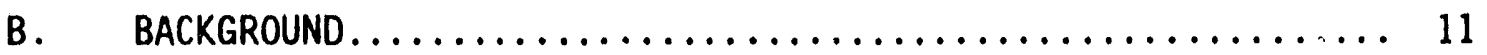

B.1 The Current Situation and Expected Future.......... 12

8.2 Fuel Availability............................. 12

B.3 Fusion Technology and Engineering............... 13

C. ECONOMIC ASSESSMENT OF FUSION POWER SYSTEMS ............. 13

D. SAFETY, ENVIRONMENTAL AND WASTE-DISPOSAL............. 14 ADVANTAGES OF FUSION POWER

E. INTERNATIONAL COLLABORATION....................... 14

F. INDUSTRY ROLES IN FUSION ENERGY DEVELOPMENT $\ldots \ldots \ldots \ldots \ldots . . \ldots$

G. UNIVERSITY ROLES IN FUSION ENERGY DEVELOPMENT $\ldots \ldots \ldots \ldots \ldots 16$

H. DEPARTMENT OF ENERGY LABORATORY ROLES IN............... 17 FUSION ENERGY DEVELOPMENT 
CHAPTER III MAGNETIC FUSION ENERGY DEVELOPMENT.................. 19

A. TECHNICAL STATUS AND PROGRESS.................... 19

A.1 U.S. Program............................. 19

A.2 Foreign Program Comparison.................... 21

B. GOALS AND OBJECTIVES .......................... 22

C. KEY PHYSICS AND TECHNOLOGY ISSUES.................. 23

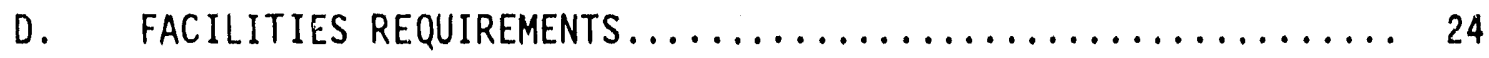

E. POSSIBLE IMPLEMENTATION STRATLGIES................. 26

E.1 Entire1y Domestic Program..................... 26

E.2 Reliance on International programs................. 26

E.3 Recommended Approach and Funding................. 26

E.4 Industrial Participation...................... 28

F. STRATEGY UNDER CONSTRAINTED BUDGETS................ 28

F.1 Near-Term Priorities......................... 28

F.2 Specific Near-Term Recommendations............... 29

F.3 Longer-term Constrained Budget Strategy............ 30

F.4 International Initiatives to Recover the Schedule..... 32

CHAPTER IV INERTIAL FUSION ENERGY DEVELOPMENT $\ldots \ldots \ldots \ldots \ldots \ldots \ldots \ldots . . \ldots$

A. INTRODUCTION................................... 39

B. CURRENT PROGRAM.............................. 40

C. KEY PHYSICS AND TECHNOLOGY ISSUES $\ldots \ldots \ldots \ldots \ldots \ldots \ldots \ldots, 41$

C.1 Target Physics............................. 41

C.2 Drivers................................... 43

C.3 Heavy Ions Drivers................................ 44

D. AlteRNATE ENERGY DRIVERS....................... 45

D.1 K.rF Laser Program........................... 45

D.2 Light Ion Program........................... 46

E. COMPLEMENTARY ENERGY PROGRAM..................... 47

E.1 Fusion Materials Development.................. 47

E.2 Reactor Design Studies............................. 47

E.3 Target Factory................................... 48

E.4 Advanced Fuels $\ldots \ldots \ldots \ldots \ldots \ldots \ldots \ldots \ldots \ldots, \ldots, \ldots$ 
F. DEFENSE AND ENERGY PROGRAM STRATEGY.............. 48

F.1 First Priority, Defense and Energy............. 49

F.2 Second Priority, Defense and Energy.............. 50

F.3 Third Priority, Energy..................... 50

F.4 Fourth Priority, Energy.................. 51

G. ORGANIZATIONAL ISSUES ...................... 51

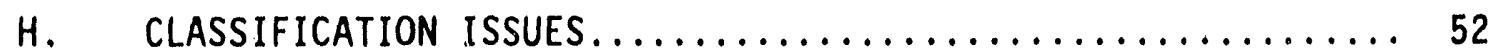

I. CONCLUDING REMARKS ........................ 53 APPENDICES :

APPENDIX 1 CHARGE TO THE COMMITTEE

APPENDIX 2 SECRETARY'S LETTER AND COMMITTEE'S RESPONSES TO QUESTIONS

APPENDIX 3 MEETINGS AND SITE VISITS

APPENDIX 4 PREVIOUS REVIEWS

APPENDIX 5 GLOSSARY 


\section{CHAPTER I}

\section{CONCLUSIONS AND RECONMENDATIONS}

\section{A. INTRODUCTION}

This document is the final report of the Fusion Policy Advisory Committee. The report conveys the Committee's views on the matters specified by the Secretary in his charge and subsequent letters to the Committee, and also satisfies the provisions of Section 7 of the Magnetic Fusion Energy Engineering Act of 1980, Public Law 96-386, which require a triennial review of the conduct of the national Magnetic Fusion Energy program. The charge to the Committee is shown in Appendix 1.

In addition to the guidance given in the formal charge, Secretary Watkins asked for an interim report, which was sent to him on July 23, 1990. The Secretary responded to this report with a July 24 letter to the Committee Chair, in which he asked a number of specific questions. The Secretary's letter and the Committee's responses to his questions are given in Appendix 2.

Three sub-Committees were established to address the large number of topics associated with fusion research and development. One considered magnetic fusion energy (MFE), a second considered inertial fusion energy (IFE), and the third considered issues common to both.

As its source of information, the full Committee read reports and heard numerous technical presentations. In addition, the sub-Committees also conducted site visits. Because U.S. fusion policy must include considerations of international fusion efforts, the committee requested and received presentations from representatives of the fusion programs of the European Community, the Soviet Union, and Japan, and from individual scientists from France, West Germany, and Japan. All meetings of the full Committee were open to the public, and public comments were solicited. Appendix 3 lists the meetings and site visits made by the Committee and sub-Committees.

For many reasons, the promise of nuclear fusion as a safe, environmentally berign, and affordable source of energy is bright. At the present state of knowledge, however, it is uncertain that this promise will become reality. Only a vigorous, well planned and well executed program of research and development will yield the needed information. The research and development process is inherently long and expensive.

The Committee recommends that the U.S. commit to a plan that will resolve this critically important issue. It also outlines the firct steps in a development process that will lead to a fusion Demonstration Power Plant by 2025 . The recommended program is aggressive, but we believe the goal is reasonable and attainable. International collaboration at a significant level is an important element ir the plan. 
Finally, the Committee outlines the priorities it would assign in the event that the recommended program cannot be fully funded. Reduced funding would lengthen the already long time horizon, and would add to the technological risk.

\section{B. THE CONTEXT OF THIS ASSESSMENT}

Issues of energy policy, such as fusion, by their nature must include other than scientific and technological considerations. The Committee believes that the context in which fusion policy must be placed today is remarkably different from that of the past. It seems very likely that the Nation's agenda in the 1990's and beyond will be strongly influenced by three issues: an increasing concern for environmental matters, the shift in national security from military capability to economic competitiveness, and renewed emphasis on a secure supply of affordable and acceptable energy. The emergence of these tipics as national issues is highly relevant, and indeed, critical to the consideration of fusion policy, and makes the context of this report fundamentally different from that of its predecessors.

The Committee believes that the United States should have a comprehensive and foresighted energy policy emphasizing the development of politically secure, safe, and environmentally acceptable sources of energy, including fusion. The events occurring in the Middle East as this report is being written underscore the necessity of a policy and commitment that survive the inevitable shortterm, unpredictable crises. The social and economic development of the United States must not be constrained by an inadequate supply of affordable energy. Yet the instability in the Middle East, the increasing petroleum imports, the environmental concerns surrounding fossil energy, and the public's reluctance to accept nuclear fission energy are alarming constraints.

Fusion research programs worldwide have made steady progress during the last decade. In the past year, tokamak test reactors in the U.S. and Europe have closely approached conditions equivalent to energy breakeven, at which point as much fusion power is produced as is required to maintain the plasma at temperature. In inertial fusion, the conditions needed for fusion energy have been bracketed between laboratory experiments using laser drivers that have approached one percent gain, and by classified experiments that have allowec demonstration of excellent performance, putting to rest fundamental questions about basic feasibility of achieving high gain. For both approaches, there is confidence that the processes occurring are sufficiently understood to take the next step toward large-scale fusion energy production.

There is an additional contextual consideration: the growing strength of foreign fusion energy programs relative to that of the U.S. In the past, the U.S. effort dominated, but this is no longer true. The magnetic fusion buidgets of ucier nations have increased, while that of the U.S. has shrunk. The European Community's magnetic fusion effort exceeds, and Japan's equals, that of the U.S. There is also increasing international interest in IFE. The committee feels strongly that international collaboration must receive greater emphasis in the formulation of U.S. fusion policy than it has in the past. Later in this report, the Committee will specifically recommend sarticipation in an international MFE project. 
Support for the fusion program has been directed primarily toward solving scientific questions. The resultant progress has been corroborated by numerous expert reviews; a selected list is given in Appendix 4. The reviews have consistently recognized gratifying progress in this difficult scientific and technological enterprise. The reviews have al so recommended that the U.S. remain a major participant in the international fusion efforts.

The Committee believes that the U.S. fusion program is now ready to initiate a goal-oriented fusion energy program. This will not be successful without major changes in the way the program is structured and funded. Development of fusion energy will be relatively expensive, and its economic return is many years away and not assured. Additionally, the availability of cheap and plentiful oil has, until quite recently, caused interest in alternative forms of energy supply to wane.

The Committee's recommendations are given in six categories: Policy, Strategy, Organization, Program and Budget, Institutional, and Management.

\section{POLICY}

The elements of the policy recommended by the Committee are:

1. The United States should commit to fusion as a potential energy source.

2. The United States fusion program must become energy oriented, with specific goals of an operating Demonstration Power Plant in 2025 and an operating Commercial Power Plant by 2040.

3. The United States should take maximum advantage of international collaboration, while maintaining sound domestic program.

4. Involvement of the private sector should be an integral part of the energy-oriented fusion program.

The Committee believes that the position established in these four policy statements is highly justified. It jelieves that the United States should be vigorousiy involved in and firmly committed to the development of fusion as a source of civilian energy, despite the high development cost and technological risk. These two factors, plus the long time required for development, are compelling reasons for government support. Other governments are increasing their fusion efforts relative to ours. If the United States is to play a significant role in this international arena, comparable commitment is essential.

There are other persuasive reasons for the Committee's strong position Fusion is one of very few energy options that offers an essentially inexhaustible and widely available fuel supply. It is the only inexhaustible energy source that has yet to be advanced to engineering feasibility. It has the prospect of boing relatively benign with respect to both worker and public safety and the production of environmentally objectionable by-products. It is now technically ready for important next steps. Research in the last few years has brought MFE 
close to the long-awaited breakeven, and significant progress has been made in IFE.

The nation will have to rely on existing energy sources-coal, natural gas, petroleum, and nuclear fission, upgraded to make them as environmentally acceptable as possible--until the transition to fusion and other energy sources can be made. A timely transition to fusion, however, will occur oniy through a disciplined, goal-oriented energy program, recommended by the Committee, and described later in this report.

The policy emphasizes the importance of a greater level of international collaboration. The program to obtain energy from fusion will be costly in terms of both human and financial resources. An aggressive component of international collaboration would both reduce each Nation's costs and focus the world's talent more sharply on the important issues.

Both MFE and IFE should increasa participation by industry. The ultimate objective of the fusion program is the commercialization of a new source of electrical energy by the private sector. The sensitive and difficult process of transferring the technology should begin now.

\section{STRATEGY}

Both MFE and IFE should be supported, although the two are at different levels of maturity, and have different technical pathways toward achieving their goals. In recommending this strategy the Committee affirms. its belief that the two approaches to civilian fusicn energy are not ready for a choice of one over the other. While MFE is now closer to achieving its scientific goals, the physical separation of driver and fusion reactor may ultimately be an important advantage for IFE. Pursuing both options at this time reduces technological risk. It is possible that a choice will take place prior to the construction of the Demonstration Power Plant, and perhaps earlier.

The strategy for both MFE and IFE is first to atiain sufficient understanding, including experimental verification of the scientific principles and phenomena related to fuel-burning plasmas and igniting pellets, then to demonstrate this understanding in a Engineering Test Facility that includes engineering and technological features, and finally, to move towards application through a Demonstration Power Plant. These demonstrations alone, however, are insufficient. There must be an independent program of concept improvement, including study, and where promising, development of alternative configurations that may be more suitable for commercialization. In addition, the overall program must include vigorous technology and materials development. Specialized fusion technology and materials development are as important to the eventual economic success of fusion as are the demonstrations of physics performance, and some of the development will require specialized facilities. 


\section{E. PROGRAM AND BUDGET RECOMMENDATIONS}

To achieve the recommended goal of one or more Demonstration Power Plants by 2025 , the total fusion energy budget, including the military ICF program and the construction of the essential facilities, would have to reach approximately $\$ 1$ billion per year in constant dollars over the period of the next seven years. Should these resources fail to materialize, the committee has developed strategies for both MFE and IFE under constrained budgets.

The program recommendations in this chapter are intended to be summaries; additional details are given in the subsequent chapters.

\section{E.1 Magnetic Fusion Energy Development}

Figure I.l shows a schedule of construction milestones and program decision points that would characterize a comprehensive MFE program. The timelines follow from the goal of having an operating Demonstration Power Plant by 2025, and a commercial Power Plant by 2040.

There is a unique opportunity to broaden international collaboration in MFE at this time. The Committee recommends a balanced strategy that takes advantage of the benefits of sharing costs, knowledge, and risk through international collaboration, while maintaining a strong U.S. program to benefit from foreign fusion research and development.

The committee recommends that the U.S. take an even-handed approach in strengthening its national and international efforts, by participating as an equal partner in the International Thermonuclear Experimental Reactor (ITER) Engineering Design Activity (EDA) and by authorizing the construction of the $U$.S. Burning Plasma Facility in the FY 1992 budget. The U.S. should propose to
locate the ITER EDA in this country, while accepting the possibility that it could be located elsewhere.

In addition to those major activities, the recommended MFE program would include a modest increase in the Base Program, D-T experiments in the TFTR, the design of a steady-state hydrogen/deuterium plasma tokamak, and increased emphasis on low-activation materials and nuclear technology. Budgets for the recommended program are shown on page 27, Chapter III.

The MFE program would meet the requirements of a budget reduced below that recommended by holding the Base Program roughly constant and stretching out the completion schedule for the burning plasma facility, while funding the $D-T$ experiments in TFTR and the ITER activity. Budgets for constrained funding levels are shown on page 31, Chapter III. The Committee recommends that international participation in the burning plasma facility be encouraged by a firm U.S. commitment to construction in FY 1992.

\section{E.2 Inertial Fusion Energy Dovelopment}

The IFE program consists of the ICF program conducted by Defense Programs, plus additional research necessary for energy applications but not needed for the defense mission. Figure I.2 presents the Committee's recommended scinedule of 
activities, construction milestones, and decision points that constitute the recommencied IFE program. The highest priority activity is the study of target physics, leading to a demonstration of fuel pellet ignition. This research is critical for both the defense mission and for the development of an IFE civilian energy option. The pellet ignition goal can probably be achieved by about the year 2000 with an early upgrade of the Nova laser to about one megajoule.

The IFE energy program must develop a driver thac operates reliably with high efficiency and iow cost, a repetition rates of few pulses per second. Heavy ion dccelerators are currently thought to be the most promising, and the Committee recommends an enhanced program for developing this technology. Because suitable heavy ion accelerators are still in an early stage of development, we recommend that two driver candidates now supported by Defense Programs, specifically k ypton-fluoride lasers and light ion accelerators, also be explored as IFE drivers. Concurrent programs should proceed in materials development; reactor design; environmental, health, and safety issues; waste disposa1; and decommissioning. The effort will complement analogous work in MFE.

The recommended budget for IFE is shown in Table II, page 51, Chapter IV. All entries are increments to the ICF budget. It is assumed that the ICF program continues to receive strong support from Defense Programs. Funding to implement the new IFE program should not be taken from existing ICF Defense Program or MFE Program budgets.

The Committee has constructed three alternative budgets, with lower levels of funding than the recommended budget. At the lowest level, the program wou'd focus almost entirely on target physics and the facilities required to obtain this information: Precision Nova, Omega and Nike. Krypton-fluoride laser research, light ion driver research, and target support would be done at a lower priority. All of these activities are funded by Defense Programs. Even at this lowest funding leve1, the Committee recommends beginning the second phase of a heavy ion driver research program and continuing the reactor studies program. The latter two items are funded by Energy Research.

The next funding increment above this lowest case would include the design and construction of the Nova Upgrade. This facility would be authorized within the next year, and construction would start in FY 1994. Meanwhile, the target physics investigations at Precision Nova and elsewhere would continue to shape the program. If the milestones were not met or if confidence in ignition at the energy provided by the Nova Upgrade decreased, construction would be delayed or cancelled.

The budget level immediately below the recommended level would support all of the activities of the two lower levels, plus an energy-funded, expanded program on the heavy ion driver, and modest activity in materials and targets.

\section{F. ORGANIZATION}

The elements that would comprise a comprehensive fusion energy program are currently spread among three organizations in DOE. The Committee recommends that two of these activities be consolidated into a new organization that 
includes the present MFE program and a new IFE component charged specifically with the responsibility for energy applications of inertial fusion. There is much to be gained from a unified administration of the two approaches. They have many issues in common.

The Inertial Confinement Fusion (ICF) program suppo ted by Defense Programs should not be a part of this organization, since its goals are distinct from those of IFE. As stated later in this report, very close coordination betren Defense Programs and the new Fusion Energy organization will be mandatory, since IFE will continue to rely heavily on the research supported froin defense purposes. The Committee recommends that the present Division of Inertial Fusion be elevated to an Office of Inertial Fusion w'thin the organization of the Deputy Assistant Secretary for Military Applications, Defense Prograns. The elements of the IFE program recommended in the subsequent section that are not supported by Defense Programs should be started at once within the current office of Fusion Energy, and not postpone 1 until changes in organization are made.

\section{G. INSTITUTIONAL ISSUES}

The ultimate objective of the recommended fusion energy program is the commercialization of a new source of electric energy. To achieve this objective the capabilities and expertise of U.S. industry, academia, and federal laboratories must be applied productively.

\section{G.1 Industrial "articipation}

In the ir energy-oriented programs, both MFE and IFE should increase participation by industry. A more formal fusion industrial participation program should be established. There is a broad range of possible relationships that can take advantage of the capabilities of industry, and would benefit both MFE and IFE in numerous ways. Effective involvement of industry would also begin the process of technology transfer.

\section{G.2 University Participation}

Buth MFE and IFE require strong participation at universities. University contributions should include education and training innovative ideas, and technological leadership in areas such as plasma physics, nuclear technology, and low-altivation materials Each participating university should have the resources to perform these functions effectively. IFE, in particular, should sponsur research at more uriversities and encourage joint efforts at major rlatiol.i: 1 facilities.

\section{G.3 Department of Energy Laboratory Partisipation}

The major new facilities that are needed for the fusion energy program wili be, to a large extent, the responsibility " $"$ " the DOE Laboratories. Development of a secure energy source from fusion power is a highly appropiate objective for these National resources. Given the necessity fur involving both the university community and U.S. industry in the process of designing, consicructing, and 
operating these facilities, the laboratories must develop more effective mechanisms to work cooperatively and share responsibility.

\section{H. MANAGEMENT ISSUES}

In addition to the institutional needs a goal-oriented and energy development focus requires attention to some program management, IFE program coordination, and classification issues.

\section{H.1 Program Management}

The Committee recommends that all fusion activities be conducted in a disciplined manner, with appropriate milestones, decision points, and downselection of options as required. The Committee further recommends that each major step in the program be subjected to a rigorous feasibility and cost analysis by a qualified external group prior to approval.

\section{H.2 Inertial Fusion Energy Program Coordination}

The IFE program should be managed to take maximum advantage of the Defense Programs research. The ICF program is presently pursuing the scientific objectives outlined in Chapter IV, and is expected to carry out the top-priority innition experiments. These experiments are the highest priority for the energy program as well. If IFE continues to look promising as a source of energy, it can be expected that a gradual separation of the IFE/ICF programs will occur, with each component remaining complementary to the other, but with different goals.

\section{H.3 Classification and Technology Transfer}

Classification policies and restrictions on transfer of fusion technology to foreign nations should be re-examined. Classification hinders the inertial fusion program. Unnecessarily restrictive policies on technology transfer hinder collaboration in both MFE and IFE. The Committee believes that classification guidelines can be written to prevent transfer of weapons technology and yet permit collaboration on the processes and targets of interest for energy applications. These changes should be made as soon as possible, before a damaging effect on public acceptance of fusion energy is caused. 


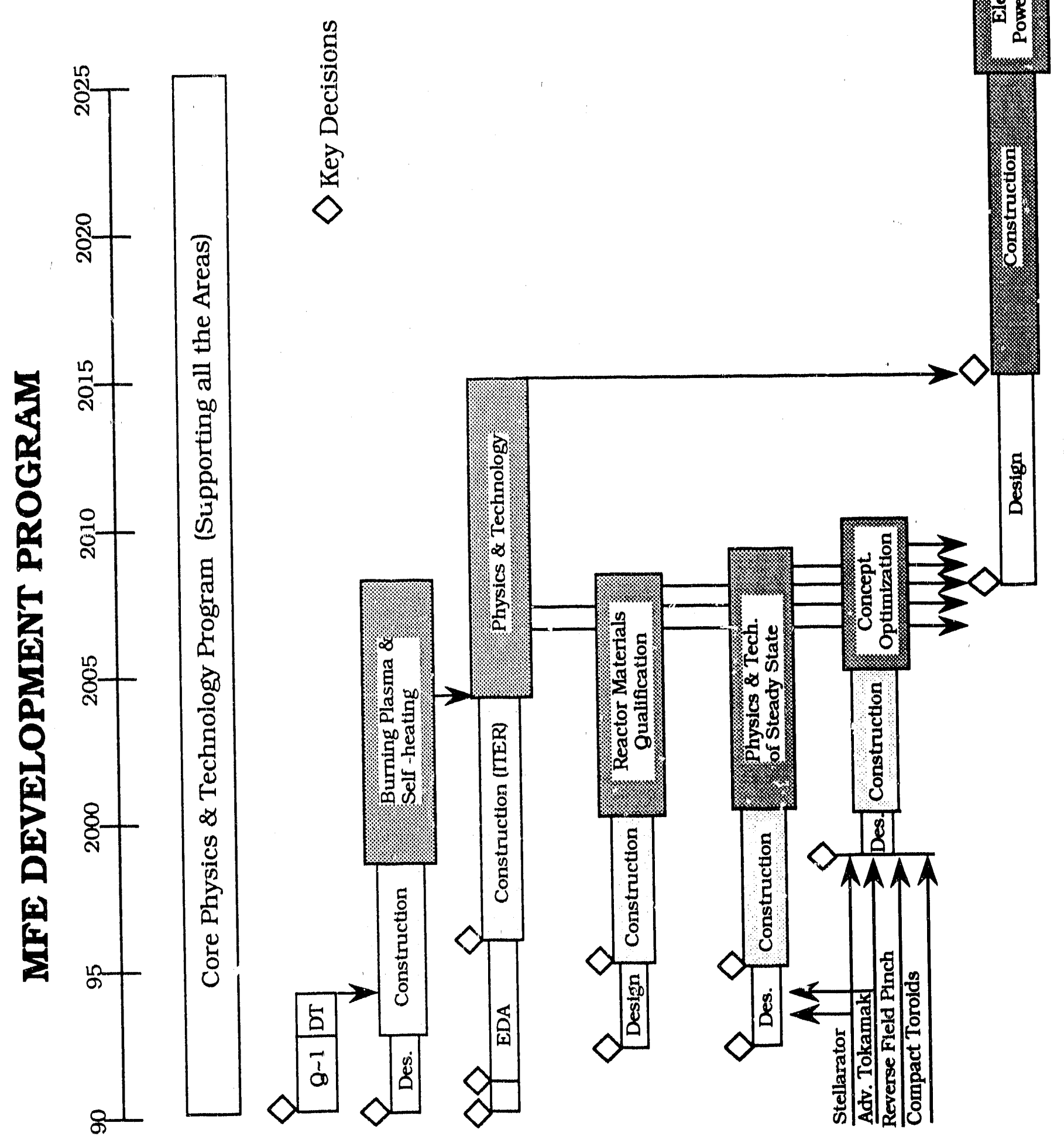

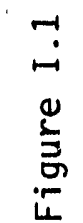

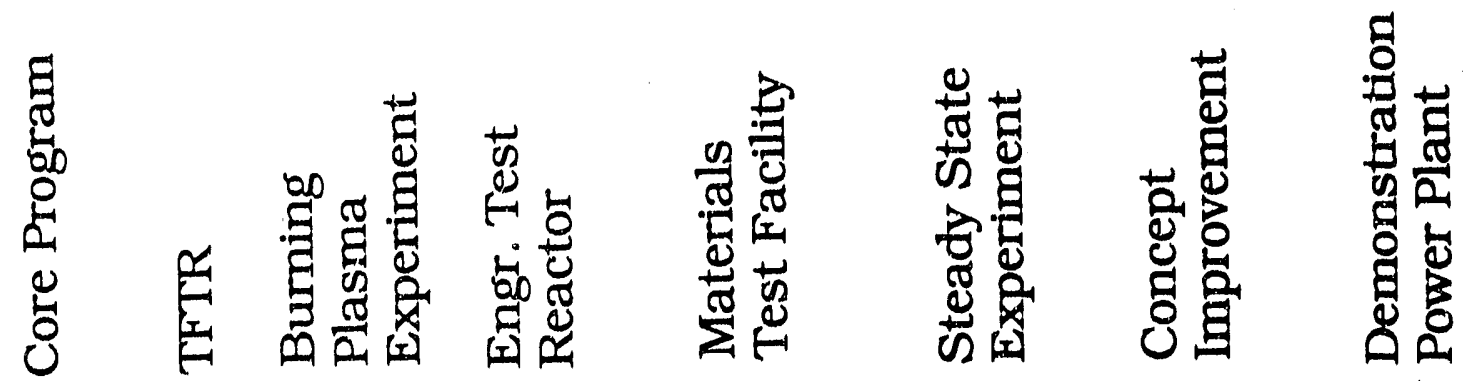




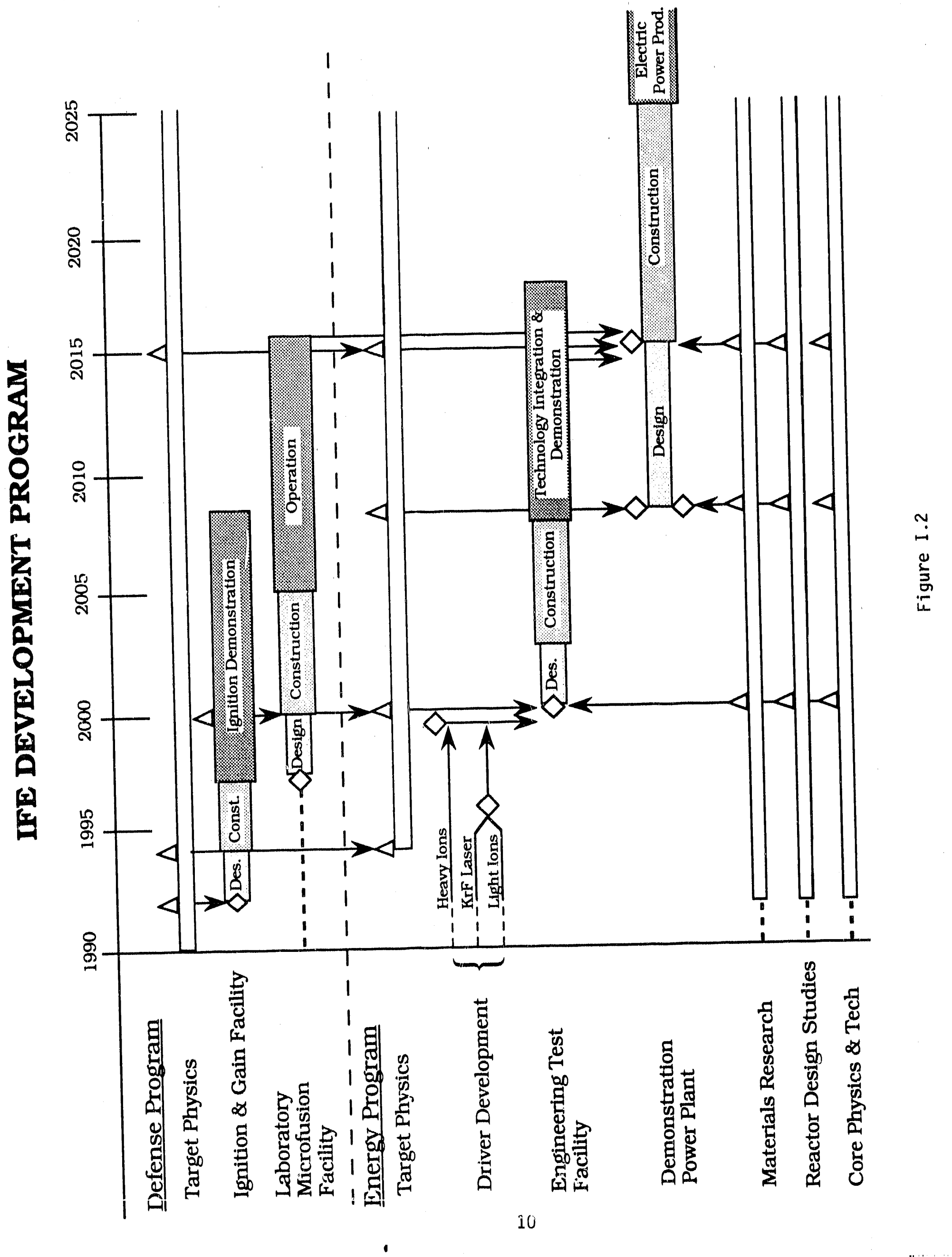




\title{
CHAPTER II
}

\author{
COMMON ISSUES
}

\section{A. INTRODUCTION}

Issues common to magnetic fusion energy and inertial fusion energy are discussed in this section. These issues include safety, environmental and waste-disposal benefits; international collaboration; and U.S. industry, university, and federal laboratory involvement. A primary incentive to develop fusion energy is to realize its potential safety, environmental and waste-disposal advantages, which, combined with its unlimited fuel supply, appear sufficient to make it competitive with other large-scale sources of energy in the long term.

\section{B. BACKGROUND}

The Fusion Policy Advisory Committe's is recommending a fusion energy strategy that provides a reasonable probability that this energy source will be available in the U.S. by the middle of the next century. This strategy is expected to culminate in at least one Demonstration Power Plant by 2025 and at least one Commercial Power Plant by 2040.

Successful implementation of this strategy requires early preparation, approval, and implementation of a detailed development-program plan with sharply defined critical paths to the first demonstration plant(s) and on to the first commercial plant(s). To be effective, this plan must have clear and logical milestones and decision points at which valid measurements of progress can be made and the direction or pace of the program changed, if necessary.

To keep the program on track, the Committee recommends that in addition to the milestones/decision-point checks, independent evaluations of progress be made periodically.

To generate as much benefit as possible for the Nation's economy from technology spinoffs and to apply the U.S. scientific, engineering and management resources as productively as possible, the recommended strategy includes strengthening the business, university, and federal laboratory infrastructure of the program, beginning immediately. Industrial personnel should be utilized not oniy in the time-honored areas of systems engineering, project management, procurement, quality control, resource allocation, equipment design and fabrication, construction, operation and maintenance, but also in areas heretofore reserved for government personnel, such as overall management, planning, scheduling and trade-off analysis. University participation must be strong enough to inject the most advanced scientific and technical knowledge into the program, to provide the best possible education and training for young people entering the program, and to carry out many basic theoretical and experimental assignments. The Department of Energy Laboratories will nave a major role in designing, constructing, and operating the large facilities that will be needed. 


\section{B.1 The Current Situation and Expected Future}

The future social and economic development of the world community requires an adequate supply of affordable energy. Widespread uncertainty about the safety, environmental compatibility, true cost, availability, adaptability and/or public acceptability of all the current major sources of energy dictates continuation of worldwide efforts to develop fusion berause it shows much potential for mitigating these concerns. Its future, too, is not yet assured. Unless a vigorous development program is maintained for the next several decades, the Nation will not have adequate hard data and experience relating to this relatively clean and safe source to make critical energy-policy decisions in the twenty-first century. These decisions include the all-important choices to be made between geopolitically neutral sources such as fusion and photovoltaics and geopolitically biased sources such as $0 i 1$.

Questions of policy, priority, and pace for the U.S. fusion-energy program must be considered in the context of preserving the global environment and protecting public health. Unrestricted industry development in the first half of this century, driven in part by the availability of cheap energy, gave rise over the past three decades to major safaty and environmental concerns: the hazards of mining and transporting coal, i.e problems of air pollution and acid rain, the prospect of global warming, and the waste-disposal aspects of fission power. During this same period, the price of fossil fuels, particularly the price of 0i1, has fluctuated widely. Political antagonism and economic cartels now are a fact of 1 ife in the oll industry. In this context, an energy policy that will ensure the development of alternative energy sources that are societally acceptable is essential. Such a policy should foster the creation of a mix of sources, including renewables and conservation, to protect against the possibility of restrictions on the use of certain technologies.

The goals established for the U.S. fusion energy program are consonant with this rationale. The characterization and validation of fusion power's safety and environmental promise must be a programmatic priority. This, combined with the establishment of a domestic technology base that validates the electric-power promise, will position the U.S. for realization of the advantages of fusion energy.

\section{B.2 Fuel Availability}

Early applications of fusion will use the deuterium-tritium reaction because it minimizes plasma containment requirements for MFE, pellet development for IFE, and temperature requirements for both. There is no practical limit to the supply of deuterium on earth, but tritium must be produced by neutron reactions with lithium. Most fusion power plants will be designed with the capability of producing tritium from lithium not only to replenish burned fuel but also for the startup of other fusion reactors. Lithium occurs in saline lakes, certain geological structures, and seawater in quantities sufficient to support a large fusion industry.

To fully attain the significant advantages of fusion energy, other reactions may be utilized in future fusion power plants, but they will require signif cant 
improvements in confinement parameters and operational limits. Examples are the deuterium-deuterium and the deuterium-helium-3 reactions.

Lack of an adequate and secure source of the helium-3 isotope is an impediment to the use of the helium reaction. Terrestrial supplies, by-product recovery from natural gas, and potential production by the nuclear-weapons complex are not large enough to be viable. Analysis of lunar surface samples indicates that this could be a significant resource, but cost estimates are inherently uncertain.

\section{B.3 Fusion Technology and Engineering}

Fusion energy embraces a wide range of nuclear technology and engineering, including topics such as large-scale superconducting magnets, materials with low-activation properties, blanket systems capable of breeding tritium and recovering the heat of fusion for conversion to electricity, plasma heating and fueling, tritium recovery and processing, nuclear performance analysis, safety and environmental engineering, and power plant design. The nuclear-technology and materials-development areas will, as much as others, determine whether the potential positive attributes of fusion will actually be realized. For this raason, the Committee has given high priority in its recommendations for both magnetic and inertial fusion energy to materials development and testing, to the need for a $14-\mathrm{MeV}$ neutron source, and to research and development on nuclear technology.

\section{ECONOMIC ASSESSMENT OF FUSION POWER SYSTEMS}

Based on several dozen economic estimates, fusion-fuel-cycle costs are expected to be less than the comparable costs of fossil-fueled and nuclear-fission plants. Total capital costs will probably be greater, however, since the construction costs associated with the heat-generating fusion core (reaction chamber, blanket shield, magnets or target drivers, and structure) are likely to be larger than the construction costs of counterpart systems in current power plants. Furthermore, most fusion concepts require that 10 percent to 20 percent of the gross electric power produced be used within the plant, which significantly increases the capital-cost burden.

These estimates are preliminary and made before the technology is fully developed. When future safety and environmental requirements are applied to existing power plants, the potential safety, waste-disposal, and environmental advantages of fusion could offset the higher capital costs.

Broad-based system studies suggest that economically competitive and environmentaliy acceptable fusion power can be obtained through a careful choice of blanket materials and reactor configurations, combined with moderate improvements in reactor materials and design. These generic investigations have been augmented by detailed conceptual-engineering studies to show specifically how fusion reactors must be designed to achieve these advantages.

In summary, numerous studies indicate that the cost of electricity generated by fusion power plants has the potential to be competitive with that generated by 
fisston or coal plants. Excluding delays and other constraints, the cost of electricity from fission or coal systems would probably be lower than that from fusion systems, as projerted today. Fusion energy appears to pose significantiy fewer safety problems and negative environmental impacts than coal or fission energy, however, and therefore is expected to achieve greater public acceptance. This may make the total life-cycle costs of fusion competitive with those of other sources in the future.

\section{SAFETY, ENVIRONMENTAL AND WASTE-DISPOSAL ADVANTAGES OF FUSION POWER}

As indicated in preceding sections of this Chapter, fusion reactors will have substantial advantages over fission reactors with respect to the consequences of severe accidents and the magnitude of radioactive-waste burdens. This is true even in the unfavorable case of fusion blankets that use non-optimal structural materials such as stainless-steel, which becomes highly radioactive under neutron bombardment. The volume of waste produced by a 1200 MWe fusion reactor, if diluted to the levels required for shallow land burial under U.S. Federal Regulations, is at least a factor of one million lower than that produced by a fission reactor of the same size. The maximum plausible critical dose at the site boundary for a severe fusion accident is two to three orders of magnitude less than that for a severe fission accident.

The disposal of waste from fusion for future designs that employ low-activation structure materials is markedly simpler than the disposal of waste from fission. The low-activation fusion reactor has roughly one millionth the radioactivity of a fission reactor at one year and about one ten-millionth 100 years after plant shutdown.

It appears that avoidance of off-site deaths from acute irradiation in severe accidents can be assured in fusion power plants without reliance on active safety systems or containment buildings. Increased experience in the last several years with the handling and control of tritium at Los Alamos suggests that fusion can meet the same high standards for routine emissions with which fission reactors now comply.

Fusion has the potential to guarantee the safety of the public by limiting radioactive-material and stored-energy inventories. No "runaway" nuclear reaction is possible. This characteristic may result in capital-cost savings by reducing the number of nuclear-stamped components relative to those required for fission power plants. For example, in a recent tokamak reactor study, the maximum plausible dose at the site boundary from a severe accident was calculated to be about $25 \mathrm{rem}$. This is the 1 imit set by regulation above which nuclearstamped components are required. The potential for achieving demonstrably sufer reactors may also reduce the time and cost associated with the licensing process.

\section{E. INTERNATIONAL COLLABORATION}

International collaboration has been an important feature of the worldwide effort to develop magnetic confinement fusion for several decades. This effort is one of the best examples in the fields of science and technology of the benefits of 
free exchange of ideas, data, concepts, personnel and equipment. Governments, National Laboratories, private companies, universities and individual consultants have all been involved. Declining budgets and lack of ne', facilities have significantly decreased the international role of the U.S. in recent years, however.

The advantages of international collaboration include: the synergistic effects of sharing knowledge and trained personnel, reduction of the financial burden for each of the participants, creation of a certain amount of human and political harmony, optimization of the use of special facilities and capabilities, increased opportunity for spincffs and the application of technical advances to related fields, and the spreading of technical and economic risks across a broad base. The disadvantages most often mentioned are a loss of secrecy, a dimicicition of economic and individual incentives, and a probable substantial increase in total combined cost over that which would be incurred by one country carrying out the project independently.

Defense secrecy is not a significant impediment to collaboration in the development of magnetic fusion. Nor is it a problem in the development of drivers for inertial fusion. It may be a problem in the development of inertial fusion targets, however, because of possible linkage to defense R\&D.

For both types of fusion the question of diminution of economic and individual incentives wollid seem to be moot at the present time. Properly balanced collaboration at this early state of the R\&D programs would provide appropriate incentives to keep all parties involved working hard to reach near-term goals. Competition for commercialization is too far out on the private-enterprise timescale to be affected by current collaboration arrangements.

With respect to the question of the effect of collaboration on total costs, the efficiencies introduced by sharing information, expertise, etc. may balance out the inefficiencies introduced by the multiplicity of participants and the melding of different techniques and procedures, etc. It is not clear at present, however, whether or not this will occur.

The committee believes that overall, the advantages of international collaboration outweigh the disadvantages. By taking a proactive role now in carefully selected international programs, the U.S. can help lead those programs into mutually productive channels.

\section{F. INDUSTRY ROLES IN FUSION ENERGY DEVELOPMENT}

The Committee recommends that, in managing the national fusion program, the Department of Energy rigorously adhere to the principle of achieving program goals with minimum integrated cost and optimum schedule. The ultimate objective of the program is the commercialization of a new source of electrical energy. Attainment of this objective would be expedited by a substantial involvement of U.S. industry, not only in the hardware phases of the program, but also in the planning, R\&D, and analytical phases. At present there is practically no private-sector participation in the program. 
The Committee therefore recommends that the Department of Energy take immediate steps to bring industry into the planning and R\&D activities already under way. This can best be done by forming carefully structured teams of federal laboratory, industry, and university personnel to handle specific assignments, by estabifshing a formal fusion industry-participation venture, and by encouraging industry-to-government and government-to-industry personnel exchanges.

The industrial-participation venture would be formalized only to the extent required by the MFE and IFE programs. It would officially encourage industrial involvement in both programs and prescribe the methods to be used to facilitate such invulvement. It would also expedite the flow of information between the Department of Energy and the various participants and provide for equitable protection of proprietary information.

A1so, an opportunity exists for industry to play a significant role in the U.S. Engineering Design Activities team in the International Thermonuclear Experimental Reactor project. This opportunity should be pursued vigorousiy. In addition, industry should be heavily represented on any other fusion-facility design teams established in either the MFE or IFE programs.

The areas of work in which industry can make important contributions have been iisted in the first section of this chapter. The many mechanisms available for involving industry on a contractual basis should be thoroughly explored and those that are beneficial to both parties should be utilized at the earliest practical date.

\section{G. UNIVERSITY ROLES IN FUSION ENERGY DEVELOPMENT}

Universities have historically made major contributions to fusion science and technology. As the fusion-energy program enters the energy-development phase, there will be an even greater need for this involvement.

Following up the comments made at the beginning of this chapter, the Committee recommends that universities:

1. Educate and train the personnel required to keep the program moving forward at an optimum pace.

2. Provide leadership in plasma physics, including theory, experimental techniques, and diagnostics.

3. Develop new and improved reactor concepts.

4. Perform complex studies and experiments in areas such as superconductors, low-activation materials, magnets, blanket:, optics and lasers, in-vessel components and materla:s, advanced plasma diagnostics and heating methods, reactor design and evaluation, and safety/risk/environmental research. 
Additional faclitities, equipment and funding are needed by the universities to perform these services. The new factlittes and equipment should be carefully chosen to effectively complement the larger, more expensive factilties and equipment avallable at the DOE Laboratories.

Untverstties have played a significant role in the development of magnetic fusion. To continue in this role, they must have strong campus-based programs and a reasonable number of larger centers carrying out an integrated, broad effort in fusion research. Further, the movement towards large-scale confinement experiments necessitates universities joining with the National Laboratories in cooperative activities centered around the large machines located at the Laboratorles. As devices at the Laboratories grow in cost, several more university confinement experiments in the \$5-\$20 million class should be inttiated. This will ensure a continued contribution of experimental plasma physics from the universities and provide a proper complement to the federal 1aboratories and industry.

With the exception of the University of Rochester, universities have played a minor role in inertial fusion. They have contributed to the theoretical understanding of laser-plasma interactions, to the experimental study of laserplasma instabilities, and to the development of fusion-reactor concepts. The inertial-fusion community would like to see universities play a more significant role. The community recognizes the importance of the intellectual stimulus, the objective critiques, and the innovative thinking provided by deeply involved untversities. Untversities should be more fully utilized in key areas such as direct-drive development, laser-plasma-interaction physics, heavy-ionaccelerator physics and design, and reactor and materials development. Specifically, the DOE should consider expanding (1) the Magnetic Fusion Energy Fellowship Program to include inertial fusion, (2) the number of universities carrying out IFE research, and (3) the number of university groups who prepare diagnostics and carry out unclassified experiments at major laboratory facilities.

Classification of certain aspects of IFE has restricted university contributions to this field. Classified research is incompatible with on-campus university activities. As the Nation moves into an inertial-fusion-energy program and revisits the classification needs as recommended by the Fusion Pollcy Advisor. Committee, the benefits that have accrued to magnetic fuston because of university involvement will accrue to inertial fusion.

Finally, university fusion-research activities will continue to provide training for people who will work in inertial fusion energy development, in fusion defense fields, in space plasma physics, and in commercial areas such as plasma processing for microelectronics manufacturing.

\section{H. DEPARTMENT OF ENERGY LABORATORY ROLES IN FUSION ENERGY DEVELOPMENT}

The Department of Energy Laboratories have played essential roles in the fusion program--through conceiving, designing, constructing, and operating a spectrum of important and innovative facllities; through generating imaginative research programs, often via the assembly of multi-disciplinary scientific and 
engineering teams; through the staffing and conduct of international activities; and by providing essential support to facility-dependent university programs.

With the recommended transition of fusion from a research to an energy program, the responsibilities of the DOE Laboratories will clearly increase. Much of the planning, construction, and operation of the major new facilities will devolve on the laboratories, as will a primary share of the responsibility for providing facilities to explore innovative concepts. The latter will require close coordination with university groups, and the Laboratories must ensure that procedures are in place to encourage and expedite such coordination. Given the necessity of involving U.S. industry in the fusion program, the DOE Laboratories and other federal laboratories must also develop more effective mechanisms to work cooperatively and share responsibility with industry. 
CHAPTER III

\section{MAGNETIC FUSION ENERGY DEVELOPMENT}

\section{A. TECHNICAL STATUS AND PROGRESS}

\section{A.1 U.S. Program}

Since the 1986 Energy Research Advisory Board review there has been signiffcant progress in extending plasma parameters to reactor conditions, increased understanding and predictability of reactor plasma regimes, advances in fusion technology, and refinement of magnetic fusion reactor concepts.

The Tokamak Fusion Test Reactor (TFTR) has increased the peak ion temperature from $20 \mathrm{keV}$ to $32 \mathrm{keV}$, and $n_{\tau} T$ from $2 \times 10^{14} \mathrm{~cm}^{-3} \mathrm{sec} \mathrm{keV} \mathrm{to} 4.3 \times 10^{14} \mathrm{~cm}^{-3} \mathrm{sec} \mathrm{keV}$ using up to $32 \mathrm{MW}$ of neutral beam heating. These deuterium plasmas produce a record $50 \mathrm{~kW}$ of fusion power and $50 \mathrm{~kJ}$ of fusion energy per pulse. The conditions project to deuterium-tritium plasmas in the breakeven regime, producing fusion power in the range of 10-30 MW and fusion energy fusion energy of 10-30 MJ per pulse. The continued progress of magnet ic fusion in producing power, and projections of fusion power for the planned D-T experiments are shown in Figure III. 1 on page 34.

In addition to producing plasma conditions approaching those of a reactor, there has been significant progress across a broad front as summarized below:

1. The key tokamak fusion power parameter $\left(n_{r} T\right)$ has increased by 10,000 over the past 15 years, and only an additional factor of seven is required to reach ignition conditions.

2. Plasma beta values of $10 \%$ have been achieved in Doublet III-D (DIII$D)$. These results are in excellent agreement with magnetohydrodynamic (MHD) theory.

3. A large experimental data base has been used to summarize and accurately predict confinement in the Low-Mode of tokamak confinement.

4. The scaling of the High-Mode of confinement has been determined by acquiring a large experimental data base, and through a detailed study using an international effort by DIII-D and the joint European Torus (JET).

5. Significant results have been obtained from the Transport Initiative including quantitative agreement between plasma transport at the edge of the Texas Experimental Tokamak and the effect of fluctuations, the correlation of $\mathrm{H}$-mode onset and plasma rotation, and the development of new fluctuation diagnostics such as beam emission spectroscopy. 
6. The prospects for steady-state current drive in a tokamak reactor were greatly enhanced by the demonstration of significant plasma current driven iotally by neutral beams of 0.5 MA on DIII-D and 1 MA on TFTR, and by the confirmation on TFTR of the theoretically predicted bootstrap current that increases current drive efficiency. Recent advanced reactor designs such as ARIES use the bootstrap effect to produce $-75 \%$ of the plasma current, thereby reducing the amount of externaliy required current drive power four-fold.

7. Several tokamak experiments, Princetion Beta Experiment-Modified (PBX-M), Versator, DIII-D, and TFTR, have produced plasma conditions near those required for second stability--a regime that could dramatically improve the tokamak reactor concept.

8. Radio frequency heating has been developed at Oak Ridge National Laboratory (ORNL) (ion cyclotron heating) and Lawrence Livermore National Laboratory (LLNL) (electron cyclotron heating) allowing high power experiments to be carried out in tokamak experiments, such as 2 MW of electron cyclotron heating on DIII-D, and $6 \mathrm{MW}$ of ion cyclotron heating on TFTR.

9. Confinement and heating properties of dense tokamak plasmas at high magnetic fields have been measured in Alcator $C$. The extensive data base from these experiments provides an essential part of the design basis for cost-effective, high-field, burning plasma experimerits.

10. Good progress has been made toward an integrated understanding of reversed field pinch (RFP) confinement through advances in both MHD and kinetic electron models, edge equilibrium and fluctuation measurements, and radial profile measurements.

11. The Advanced Toro \&l Facility (ATF) stellarator, an inherently steady state concepi, has been operated with plasma confinement comparable to tokamaks of similar dimensions. Experiments on ATF have confirmed physics expectations for a second region of stability and for cootstrap current. These effects share many overlapping physics issues with tokamaks.

12. The implementation of wall coating techniques (carbonization and boronization) and carbon/carbon limiters has increased the performance of TFTR and DIII-D. Advanced divertors using electrical biasing techniques have been designed by UCLA, DIII-D and PBX-M.

13. Pneumatic pellet injectors developed at ORNL enabled TFTR to achieve record $n_{\tau}-1.5 \times 10^{14} \mathrm{~cm}^{-3} \mathrm{sec}$ - which is equal to the minimum $n_{r}$ required for ignition of a D-T plasma with flat profiles.

14. The Tritium System Test Assembly at Los Alamos National Laboratory (LANL) has achieved an excellent safety record through seventy-two months of tritium operations, processing tritium at the rate of over $1 \mathrm{~kg}$ per day through the plasma exhaust gas processing loop. 
15. The Large Coil Project at ORNL was successfully completed with operation at or above the design magnetic field for all coils.

16. A data base on radiation effects in austenitic steels has been developed at ORNL.

17. Significant contributions have been made to the U.S. science and technology base in supercomputers, gyrotrons, superconducting magnets, atomic physics, the Strategic Defense Initiative and plasma material processing.

18. The International Thermonuclear Experimental Reactor (ITER) Conceptual Design Activity has demonstrated successful international collaboration in this large science project.

Figures III.2 and III.3 on pages 35 and 36 put some of these results in perspective with the various needs of the program by comparing the goals and approaches of the principal facilities contributing to tokamak and alternative concept physics issues. These figures exhibit the complementary nature of the international program and the need for a burning plasma experiment to study alpha particle physics in the U.S.

\section{A.2 Foreign Program Comparisons}

The Joint European Torus (JET), the largest tokamak in the world, operates with plasma currents up to $7 \mathrm{MA}$, ion cyciotron radio-frequency (ICRF) heating up to $16 \mathrm{MW}$ and neutral beam heating up to $18 \mathrm{MW}$. JET has produced reactor level plasma parameters (not simultaneous ly) of $T_{i} \sim 25 \mathrm{keV}, n \sim 4 \times 10^{14} \mathrm{~cm}^{-3}$ and $\tau$ 1.8 seconds. Using beryllium coatings on the vacuum vessel wall, JET has obtained deuterium fusion power yields of $40 \mathrm{~kW}$ and $40 \mathrm{~kJ}$ per pulse. The JET plasma conditions project to $0-0.7$ in D-T plasmas. The Tore Supra, a superconducting toroidal field coil tokamak, has begun operation in France, along with a superconducting toroidal field coil tokamak, T-15, in the Soviet Union. The ASDEX tokamak in Germany continued to provide advances in enhanced confinement and current drive, and will soon be replaced by ASDEX Upgrade, a 2 MA tokamak dedicated to studying plasma-wall interactions. In Japan lowerhybrid heating was used to drive piasma currents of 1.5 MA in the large tokamak (JT-60) and, in the smaller Triam superconducting tokamak, lower hy- d sustained the discharge in steady state for more than 1 hour. JT.-60 is currently being upgraded to have an ovarall capability comparable to JET. The T-10 tokamak in the Soviet Union has been the world leader in ECH, producing power of $-2 \mathrm{MW}$ and electron temperatures of $\sim 10 \mathrm{keV}$.

Both the European Commurity (EC) and Japan have operating tokamaks which are substantially larger and more E’pensive than the largest U.S. device (TFTR). Furthermore, both have funded major upgrades of their principal experiments.unlike the U.S. All three foreign parties to the ITER discussions have operating superconducting tokamaks with the U.S. only now beginning to plan for such a device in the late 1990's. Thus, while the U.S. has contributed significantly to world progress on tokamaks in the $1980^{\prime} \mathrm{s}$, it will fall behind in the $1990^{\prime} \mathrm{s}$ unless new investments are made, as recommended later in this ieport. 
In the case of alternative concepts, the situation has fluctuated. The U.S. was behind in the early $1980^{\prime} \mathrm{s}$. Now with new stellarator, reversed field pinch, and compact torus devices--which have been delayed and underutilized due to budget cuts--the U.S. has the potential to lead for most of this decade. Then, based on EC and Japanese plans for new and larger devices, the lead is expected to shift away from the U.S. in the mid 1990's. This is healthy and all parties will benefit from the new and better data to enable a choice between the tokamak and one of these concepts as the basis for the Demonstration Power Plant. However, it does point out the importance of existing collaboration and the broader data base in physics understanding provided by these approaches.

The U.S. has provided hardware and scientific manpower for joint experiments on foreign facilities such as JET, Tore Supra, ASDEX and TEXTOR in Europe and smaller facilities in Japan. Similariy the Japanese and European programs have made substantial investments in materials and manpower applied to experiments in the U.S., notab7y on DIII-D, ISX-B, MTX, the Tritium System Test Assembly at Los Alamos, and the international Large Coil Project at Oak Ridge National Laboratory. These joint ventures, inside and outside the U.S., have established a positive history of balanced collaboration for mutual benefit.

\section{B. GOALS AND OBJECTIVES}

Based on decades of steady progress worldwide, the Committee believes that the magnetic fusion program is ready to meet the challenge of preparing for an operating Demonstration Power Plant by 2025. As noted above, recently the program has achieved, in the TFTR at Princeton and in the JET in Europe, the parameters in deuterium plasmas that would approximately produce "breakeven" with deuterium and tritium fuel. In pure deuterium, fusion power production of - $50 \mathrm{~kW}$ has been achieved, about $50 \mathrm{~kJ}$ per pulse. With tritium, these devices would produce more than $10 \mathrm{MW}$ of fusion power. Experiments with tritium can be carried out in TFTR in 1993-1994.

The present results meet the original goals of facilities designed in the early $1970^{\prime} \mathrm{s}$ based on the scientific understanding and empirical database then available. Meanwhile, many more reactor design features have been well enough understood and experimentally verified to be reduced to straightforward engineering design calculations. An empirical database has been accumulated on the rate of heat transport that is sufficiently reliable to predict the critical size of a device for high power gain and ignition within reasonable bolinds of uncertainty; and the program is solidly focused on identifying and quantifying the turbuience processes that govern trarisport (the Transport Initiative).

Thus, there now exists the scientific basis to proceed with a burning plasma experiment that could produce at least $100 \mathrm{MW}$ of fusion powf:r and a gain factor, $Q$, of 5 or more by the year 2000. Such a device, operating at magnetic fields around 9 Tesla, would provide reactor physics data with burning plasmas in the high field configuration presently believed most promising for the Demonstration Power Plant. As the premier experiment at the turn of the century, it would al so make the U.S. a strong and attractive partner in magnetic fusion research. 
The present data base also provides confidence to design a tokamak fusion reactor that would address the engineering feasibility of fusion. Such a conceptual design, the International Thermonuclear Experimental Reactor (ITER), has been completed this year by an international consortium including the U.S., U.S.S.R., Japan and the EC. FPAC heard presentations by senior representatives from the U.S.S.R., Japan and the EC who expressed confidence in the technical basis for ITER and a readiness to proceed to the engineering design phase of this project.

With this background, the Committee recommends that the magnetic fusion program clearly focus on its energy mission by adopting the goal of an operating, environmentally attractive Demonstration Power Plant by 2025.

If there were an assured supply of Helium-3, burning advanced fuels could lead to even more attractive reactors. This would require that the difficult confinement and heating problems associated with the D-Helium-3 reaction be solved. Reducing the neutron flux and eliminating blanket breeding of tritium simplifies considerably the materials and radiological problems of fusion.

\section{KEY PHYSICS AND TECHNOLOGY I SSUES}

The successful achievement of the goals recommended by the Committee requires research and development in magnetic fusion aimed at the resolution of key physics and technology issues. The key physics issues include:

Confinement: To increase understanding of the phenomena which dominate degradation in confinement.

Power handling and particle exhaust: To determine ways for reducing to acceptable levels the power load on the most exposed components, and to develop improved means of controlling impurities and exhausting particles under reactor conditions.

Plasma heating, fueling and non-inductive current drive: To develop heating and fueling methods for high power plasmas and methods for driving plasma current efficiently.

Alpha particle heating: To understand how burning plasmas behave under conditions of dominant self-heating by the alpha particles produced in fusion reactions. As no tokamak has yet operated in this mode, the physics of plasmas heated by alpha particles is thus far known only through theoretical models and numerical simulations.

Operational limits and disruption: To better understand the operational limits on plasma pressure, current, and density.

Alternative concept physics: To achieve substantial progress in plasma parameters and to investigate plasma performance of stellarator and RFP experiments. 
The key technology issues to be addressed include:

Blankets for heat transfer, tritium breeding, and shielding: To develop blankets which are suitable for fuel production (tritium), energy extraction and radiation protection.

Heating and fueling systems: To develop the requist te plasma heating and fueling technology for a fusion reactor.

Large, high field superconducting magnets: To develop magnets of the size, field, current, and reliability required for reactor-size devices.

Materials: To develop structural, first wall, and blanket materials, including low activation materials, that would enhance fusion's economic and environmental characteristics.

Remote handling and maintenance methods: To develop adequate remote handling and maintenance methods to maximize fusion reactor availability and minimize risk to maintenance personnel.

Safety arid environment: To minimize the consequences of severe accidents and waste disposal.

The existing U.S. and foreign facilities are currently contributing to the understanding of many of the key issues. A chart describing the expected contributions of the major facilities ( $p$ lasma current $>1$ MA) through the mid $1990^{\prime} \mathrm{s}$ is shown in Figure III.4 on page 36. These facilities along with the smaller facilities are making steady progress as described earlier, but there is a clear need for newer, more capable facilities. The continuing exploitation of existing factlities and the con truction of new, more capable facilities should provide for the resolution of the key physics and technology issues necessary for construction of a Demonstration Power Plant on the schedule indicated.

Figure I.1 on page 9 shows the Committee's recommendations for a schedule of construction milestones and program decision points for facilities that would be necessary to address the key physics and technology issues. These facilities form the basis of the MFE technical development program. The timelines shown represent an orderly, sequential program, and follow from the goal of having an operating Demonstration Power Plant by 2025, and a Commercial Power Plant by 2040.

\section{FACILITIES REQUIREMENTS}

The recommended MFE program plan includes four major new facilities to be initiated in this decade. Each of these facilities. would be candidates for varying degrees of international collaboration. These facilities are: 
1. Burning Plasma Facility, e.g., the Compact Ignition Tokamak (CIT) ${ }^{1}$

2. Engineering Test Facility, e.g., the International Thermonuclear Experimenta1 Reactor (ITER) ${ }^{1}$

3. Steady-state hydrogen/deuterium - plasma tokamak

4. $14 \mathrm{MeV}$ neutron source for materials development and testing (which serves both MFE and IFE needs).

The major expenditures are for the high magnetic field, low current, Blirning Plasma Experimerit (CIT), and an Engineering Test Facility (ITER). ITER will probably be a lower magnetic field, high current device. Its design, done internationally, will incorporate the new information that becomes available from CIT and from other participants in the international fusion research program. These two new facilities will be the pioneers entering the new regime of burning plasmas at high power gain.

The Committee believes that both CIT and ITER are essential to proceed fito the 1990 's with the confidence to meet our stated goal of an operating Demonstration Power Plant by 2025. These factlities are complementary. The goal of ITER is to produce about 1000 . MW of fusion power at high gain $(Q>5)$ in long pulses, ultimately steady state. Once operational, ITER would be used for engineering testing including nuclear power components. The CIT would produce $100 \mathrm{MW}$ or more of fusion power, also at high gain $(Q>5)$, but in pulses of about 10 seconds in duration. The smaller CIT could operate several years before ITER and provide valuable input on burning plasmas prior to ITER operation. This follows the successful development strategy of the past in which the smaller Princeton Large Torus developed neutral beam heating for the TFTR, then in construction; ASDEX revealed the $\mathrm{H}$-mode of operation that ultimately doubled the performance of JET; and small U.S. facilities developed the lower-hybrid current drive now employed successfully on the Japarese JT-60. Similarly, CIT could provide advanced information that would avoid a prolonged arid costly startup Tearning period in ITER.

The achievement of $100 \mathrm{MW}$ of fusion power in CIT would be a milestone achievement in its own right, intermediate between existing facilities and ITER. A]so, the CIT, using high-field copper magnet technology and thus limited to short pulse operation, would provide pioneering data on reactor core physics at the high fields that should become technically feasible for long pulse superconducting magnets by the time the Demonstration Power Plant is constructed. High field reduces capital cost and improves performance.

\footnotetext{
'In this report, the terms Burning Plasma Experiment and Compact Ignition Tokamak (CIT) are used interchangeably. The use of the term CIT is generic, and does not imply a specific design or location for this facility. The Committee understands that the current CIT design is being reviewed, to ensure that it meets the objectives stated in this report. Similarly, the terms Engineering Test Facility and ITER are used interchangeably, and do not imply a specific design or location for this facility.
} 


\section{E. POSSIBLE IMPLEMENTATION STRATEGIES}

\section{E.1 Entirely Domestic Program}

The technical development program described above could be pursued by the U.S. independentiy. Such an implementation strategy would require that the U.S. construct and operate each of the facllities in the recommended technical development program. Although international cooperation would probably cont inue at some level, the U.S. MFE program would not depend on foreign programs. The U.S. could possibiy regain the position of world leader in fusion research and would be a highly desirable partner for collaboration.

The primary disadvantage to this approach would be the cost, particularly of an ITER-class U.S. Engineering Test Facility that would be the first device to significantly exceed the cost of existing fusion devices. This strategy would fail to take full advantage of the intellectual and financial resources devoted to fusion research abroad and would require substantial funding increases reaching total funding levels in excess of one billiton dollars a year. Nevertheless, the ongoing collaboration and international exchange of data would assure that fundamental mistakes were not made. The strong U.S. program assumed here would also assure that American industries are fully up to speed for their future competitive role. The committee concludes that the advantages of the entirely domestic development program are outweighed by the cost.

\section{E.2 Reliance on International Programs}

The Committee has also evaluated the strategy of relying entirely on the other major world fusion programs for the construction and operation of the major new facilities. The advantage in this approach would be the avoided costs. The disadvantages of this approach are numerous: The U.S. would lose control over determining the viability of magnetic fusion energy; there would be considerable damage to the U.S. fusion infrastructure, U.S. industries would fall behind their international competitors, and the U.S. would no longer be considered a desirable partner in collaborative fusion research. The U.S. would be at a significant disadvantage should fusion technical development succeed and future circumstances favor fusion as an energy supply option. Therefore, the Committee rejects this option.

\section{E.3 Recommended Approach and Funding}

The Committee recommends a balanced strategy that takes advantage of the benefits of sharing costs, knowledge, and risk through international collahoration while maintaining a strong U.S. program able to benefit from foreign fusion research and development. The strategy would build on the major, well-cstablished international collaborations between the U.S. and the major fusion programs in Japan, the Soviet Union, and the European Community. To date, these collaborations have yielded numerous examples of mutual benefit.

In the technical development program described in Section $D$, the Engineering iast Facility and the $14 \mathrm{MeV}$ neutron source, since they are both test facilities is at all parties could utilize for their own purposes, are ideal candidates for international management and cost sharing. 
We strongly recommend that the U.S. participate as an equal partner in the ITER Engineering Design Activity and that the U.S. propose to locate the EDA in the U.S. We also recommend that the U.S. take the lead in organizing international collaboration on the $14 \mathrm{MeV}$ neutron source. Active U.S. participation in these international endeavors will ensure that U.S. views continue to be heard as these projects evolve. Despite the added complexity of international agreements, we believe these recommendations can be made consistent with the goal of an operating Demonstration Power Plant by 2025.

The committee also recommends construction as soon as posstble of the U.S. Burning Plasma Facllity. To be a desirable partner in ITER and other international collaborations and to benefit from the results of the research, the U.S. must maintain a strong tomestic program including proportionate contributions to world progress. As the world's premier magnetic confinement facility in the period preceding ITER operation, a U.S. Burning Plasma Facility producing $100 \mathrm{MW}$ of fusion power would be an exciting achievement and reestablish U.S. leadership in the field.

Ful1 implementation of the recommended approach requires substantial increases in funding. An example five-year budget, based on input to the Committee, is (constant FY 1990 dollars in millions):

TABLE I

FY 90

Actual

$\underline{9}$

Base Program

285

325

33

ITER/CIT Conceptual Design

ITER Engineering Design Activity

CIT Construction



$\underline{55}$

40

40

60

60

318

420

475

170

$180 \quad 175$

318

570

$590 \quad 620$

Here the Base Program includes present activities in plasma science and technology development plus about $\$ 175 \mathrm{M}$ per year activity on existing major tokamak and advanced toroidal facilities. The indicated increases in the Base Program provide first for D-T operation in TFTR, as indicated in the Development Plan, and also for more efficient use of other underfunded facilities. Then, as TFTR and other facilities are retired later in this decade, residual funds would be used to begin design and construction of two new facilities addressing remaining problems within the Base Program. These are the steady-state hydrogen/deuterium plasma tokamak, which is the most important next step in tokamak concept improvement and an important support facility for ITER, and the $14 \mathrm{MeV}$ neutron source for materials development. 
The recommended Base Program implies dynamic changes and difficult cholces for the program over the next four years. Following this plan, the existing major tokamak factitties would be retired in the second half of this decade. This implies dislocation of personnel, an uncertain future for institutions, and the transfer of reusable equipment to whichever site is chosen for the new steadystate factlity. Similar dislocations will occur as smaller advanced toroldal factlities, some still in construction, are retired and a down-selection among alternative concepts is made later in the decade. However, there is generai agreement in the community that these sacrifices must be made to make way for new initiatives. To minimize their disruptive effects, these changes should be managed to conserve as far as possible the present scientific and engineering talent in laboratories, universities and industry.

To put these activities and needs into perspective, it is instructive to view the funding history of the U.S. MFE program. Figure III.5 on page 38 shows the annual funding level since its inception in 1990 dollars. The increases needed to participate in ITER and construct CIT are modest compared to those driven by the energy crisis of the early 1970's.

\section{E.4 Industrial Participation}

Industrial involvement should increase significantiy through involvement in ITER and new facility construction. The range of possible activities includes exchange or loan of personnel, professional-service contracts, purchases of equipment and systems, and construction/maintenance/operating contracts. Increased industrial involvement will benefit the program in several ways: broadened technical support; improved programs and project management; expert assistance in procurement; quality control; resource allocation; and enhancement of capability for equipment fabrication, facility construction, and plant operation and maintenance. It will also begin the process of technology transfer to industry.

\section{F. STRATEGY UNDER CONSTRAINED BUDGETS}

The Cummittee believes that there are compelling reasons for the timely development of fusion energy that justify the required increases in funding described above. Even if these necessary increases are not immediately forthcoming, we nonetheless recommend starting down the developmerit path we have outlined, with the following priorities and strategy for stretching out the schedule.

\section{F.1 Near-Term Priorities}

The Committee has extensively explored the question of the relative priority in the near term of the international Engineering Test Facility and of the U.S. Burning Plasma Experiment. We have come to the firm conclusion that a strategy which selects either one of these program elements over the otiner would certainly be unwise both for the U.S. fusion effort, and also for the world program. A CIT-only strategy leaves the U.S. out of an international col?aboration whose value extends beyond the key technical one of participating $i$ the construction and operation of a fusion Engineering Test Reactor. An 
ITER-only strategy, on the other hand, leaves unanswered the central sctentffic questions of burning plasma physics for over 15 years, and falls to restore the U.S. fuston program to parity with its major foretgn competitors. A weak U.S. program would iimit American influence on the ITER project, and would also compromise the abillty of the U.S. fuston infrastructure to capttalize on what is learned from ITER construction and operation.

The ITER project has identifted a 1ist of physics and engineering R\&D issues that need to be resolved in order to support a decision for construction in 1996; their resolution will influence the Ultimate ITER design and schedule. Irrespective of the evolution of the ITER program, a CIT Burning Plasma Experiment under construction at that time puts the U.S. and the world fusion effort in a strong position to demonstrate significant production of fuston power, and to answer many key scientific questions about burning plasma physics, at about the turn of the century. If ITER construction does go forward on its currently projected schedule, research results from CIT will greatiy reduce the risk that ITER could run into difficulties which would comprointse tts ETR mission.

Based upon these considerations, we conclude that even under a constratned budget the U.S. should take an even-handed approach to strengthening its national and international efforts in magnetic fuston research. That is, the U.S. should both conduct its own burning plasma experiment, CIT, and also play a prominent role in the international engineering test reactor. As first steps, we recommend that, even with a constrained total budget, the FY 1992 funding for the ITER Engineering Design Activity and for the CIT Burning Plasma Experiment each be increased to $\$ 40 \mathrm{M}$ from their FY 1991 levels of $\$ 16 \mathrm{M}$ and $\$ 17 \mathrm{M}$ respectively, and that a Burning Plasma Expertment be authorized as a construction line item in the FY 1992 budget.

As regards existing facilities, the most important question is the best use of the Princeton TFTR facility, which is currently the major U.S. asset. The Committee recommends that Princeton proceed expeditiously to carry out D-T experiments in TFTR. These experiments will provide the world's first information on alpha particle behavior in burning plasma's, and give assurance against the possibility that confinement deteriorates severely when burning occurs. Highest priority in D-T operation should be given to these narrow physics objectives in order to limit activation of the machine and the cost of its decommissioning. According to the information piovided to the committee, such limited D-T operation does not preclude future usage of the TFTR bulldings and certain auxiliary equipment for either a follow-on CIT or a steady-state advanced hydrogen/deuterium tokamak.

\section{F.2 Specific Near-Term Recommendations}

With this background, we make the following recommendations:

1. Participate as an equal partner in the ITER Engineering Design Activity (EDA), with major participation by U.S, industry, and propose to locate this activity in the U.S. An increase in the MFE budget should be provided for this purpose, bringing the budget for ITER to $\$ 40 M$ in FY 1992. 
2. Commit to construction of CIT and inftiate the engineering design effort in FY 1992 with the CIT budget increased to $\$ 40 \mathrm{M}$.

3. In the Base Program

a. Carry out: D-T experiments in TFTR as expeditiously as possible. In this regard, DOE should reviow the most recent environimental protection requirements and their cost implications.

b. Provide a modest funding increase to improve the productivity of other confinement factlittes. Cont inue ongoing tokamak and alternative concept programs to a productive conclusion.

c. Inttiate design of the steady-state advanced hydrogen/ deuterlum tokamak facility included in the development plan.

d. Increase funding for the development of nuclear iechnology and low-activation matertals, and move toward an international plan to construct a $14 \mathrm{MeV}$ neutron source.

\section{F.3 Longer-Term Constrained-Budget Strategy}

In addition to the strategy of selecting between CIT or ITER, considered and rejected in the previous section, the committee also considered and rejected other strategies as follows:

1. Accelerate CIT by stopping TFTR early. We rejected this because we place high priority on D-T results from TFTR at the earliest possible time.

2. Stop other Base Program activities to fund ITER and CIT. We rejected this because of the value of these programs, and the waste in abandoning important and highly productive equipment already built.

3. Reduce the cost of the Burning Plasma Experiment. We rejected this because, based on the information provided to us, we believe that a high gain Burning Plasma Experiment in the $\$ 1$ B class incorporating a divertor (known to improve confinement) and superior diagnostic capability is the prudent course.

With this background, we have constructed a "Constrained Budget" scenario which provides for D-T experiments in TFTR and a modest increment to the Base Program in the near term. It delays the new initlatives in the Base Program included in the Recommended Budget unt 11 the mid-1990's when funds will beg in to be freed up by the completion of some present programs. This Constrained Budget scemarto provides the full projected budgets required for the ITER EDA, as we know them; the actual ITER budgets will be a matter for detafled international negotiation. Under this budget scenario, the CIT Burning Plasma Experiment is committed in FY 1992, but construction is stretched out to two years relative to the 
Recommended Budget. A delay of first plasma in CIT would be unfortunate, but since the complex ITER process is itself likely to involve some stretchout and delay, wa do not feel that this results in a qualttattve change in the role of the CIT Burning Plasma Expertment in the U.S, and world programs. The Constralned Budget scenarto is given below, as well as the total Recommended Budget, for compartson (in constant milltons of FY 1990 dollars).

\section{TABLE II}

$\begin{array}{lcrrrrr} & \begin{array}{c}\text { FY } 90 \\ \text { Actual }\end{array} & 92 & 93 & 94 & 95 & 96 \\ \text { TFTR } & 60 & 75 & 75 & 65 & 20 * & 20 * \\ \text { Other Base } & 225 & 230 & 230 & 230 & 230 & 230 \\ \text { ITER } & 16 & 40 & 40 & 50 & 60 & 60 \\ \text { CIT } & 17 & 40 & 90 & 105 & 160 & 160\end{array}$

Constrained Budget:

$\begin{array}{llllll}318 & 385 & 435 & 450 & 470 & 470\end{array}$

$25 \%$ International Support for CIT:

$\begin{array}{llllll}318 & 385 & 413 & 424 & 430 & 430\end{array}$

For Compartson Recommended Budget: (Section E.3)

$\begin{array}{llllll}318 & 420 & 475 & 572 & 590 & 620\end{array}$

*Decommisstoning costs after D-T experiments are completed in FY 1994.

The Constrained Budget can be further reduced, or the delay in CIT can be recovered, if modest (25\%) international participation in CIT can be developed starting in FY 1993, after an inftlal U.S. commitment in FY 1992. This scenarto is inclurled in the table and discussed further in Section F.4 below.

Note that the Constrained Budget over five years builds the program to approximate parity with the present European Community funding level of 450 million ECU per year. This higher level of funding has permitted Europe to continue to invest in techrology development and major factitties in the years when U.S. fusion funding was sharply declining (see Section A.2). By committing to CIT construction, the U.S. now has the opportunity to regatn leadership in the 1990's with technical results that both complement ITER and provide a major milestone in fusion research. 
It must be recognized, however, that the Constrained Budget, in stretching out CIT construction and delaying the initiation of the recommended new initiatives in the Base Program, is likely to push bock the operating date for the Demonstration Power Plant beyond 2025.

\section{F.4 International Inttiatives to Recover the Schedule}

The development schedule under a Constrained Budget could be shortened and outyear costs reduced if the highly successful collaborative effort on ITER were extended to include other program elements such as CIT, the $14 \mathrm{MeV}$ neutron source, the steady-state advanced hydrogen/deuterlum tokamak and alternative concepts.

We strongly recommend that the U.S. play a leading role in negotiating a coordinated world effort centered on the design and construction of ITER and international collaboration on supporting programs. A commitment to CIT in

FY 1992. and initiation of design and planning of the steady-state advanced hydrogen/deuterfum tokamak and $14 \mathrm{MeV}$ neutron source, as recommended in Section F.2, will greatly strengthen the U.S. position in these negotiations.

Supporting programs can be organized bllaterally or multilaterally, depending on the interests of the partners. As an example, consider the following division of effort on the major facilities we have recommended in addition to ITER:

TABLE III

$\begin{array}{ll}\text { U.S. Alone } & \text { U.S. Costs } \\ \text { W/Partners }\end{array}$

CIT (if $25 \%$ international)

$\$ 1000 M$

$\$ 750 M$

Steady-State Tokamak ( 2 partners assumed)

500 (approx.)

250

$14 \mathrm{MeV}$ Neutron Source

300 (approx.)

75

(4 partners assumed)

Total Capital Investment

$\$ 1800 M$


For this example, since U.S. costs for all three factlittes constructed internationally would be about the same as CIT alone, these costs could be distributed within the same budget profile as that for the Constrained Budget. Moreover, through continued international collaboration in the operation of these facllities, the budget could remain at approximately the $\$ 500 \mathrm{M}$ per year level into the late $1990^{\prime} \mathrm{s}$ using the roll-off from CIT construction and the other programs to fund the construction of ITER. 


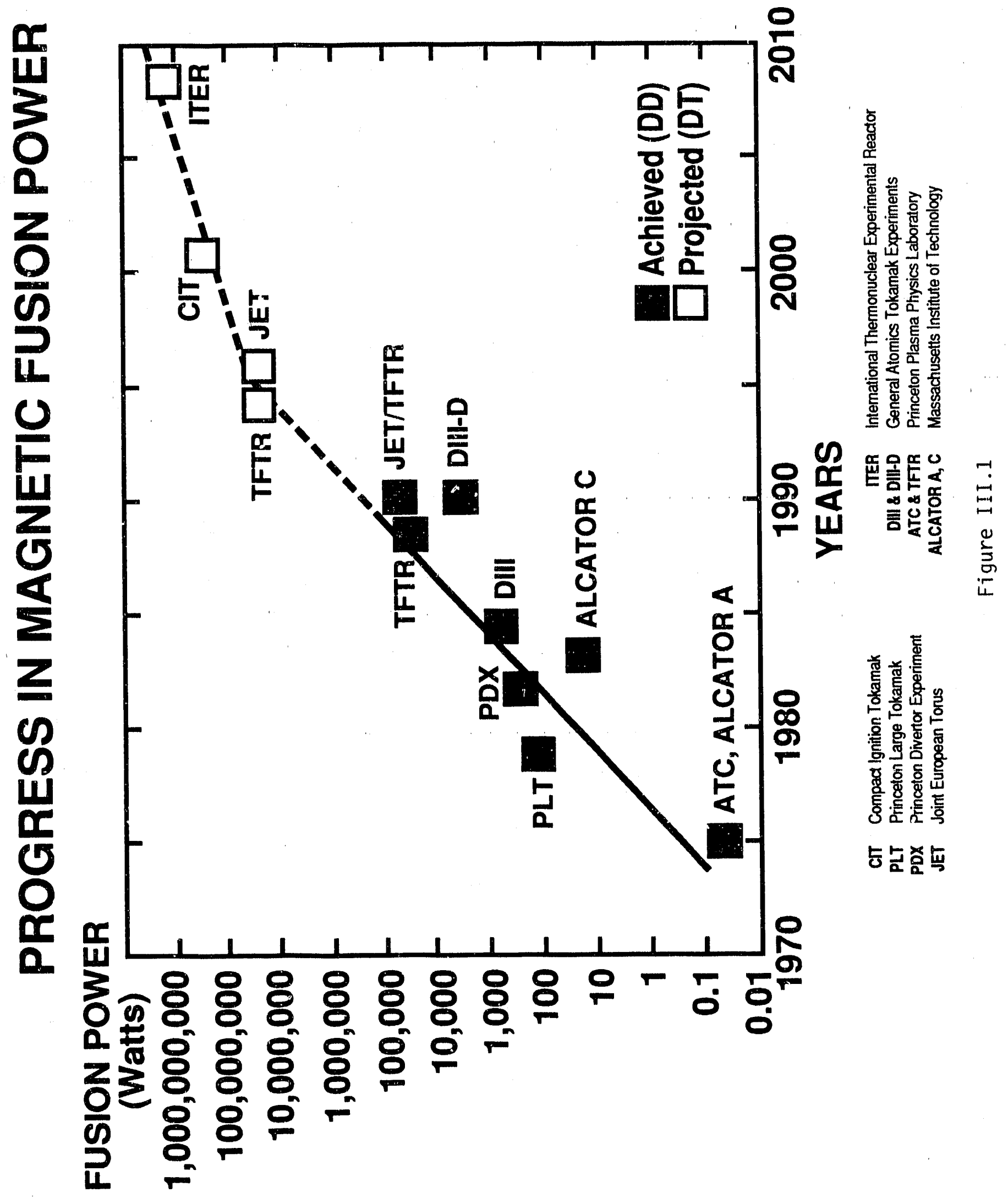




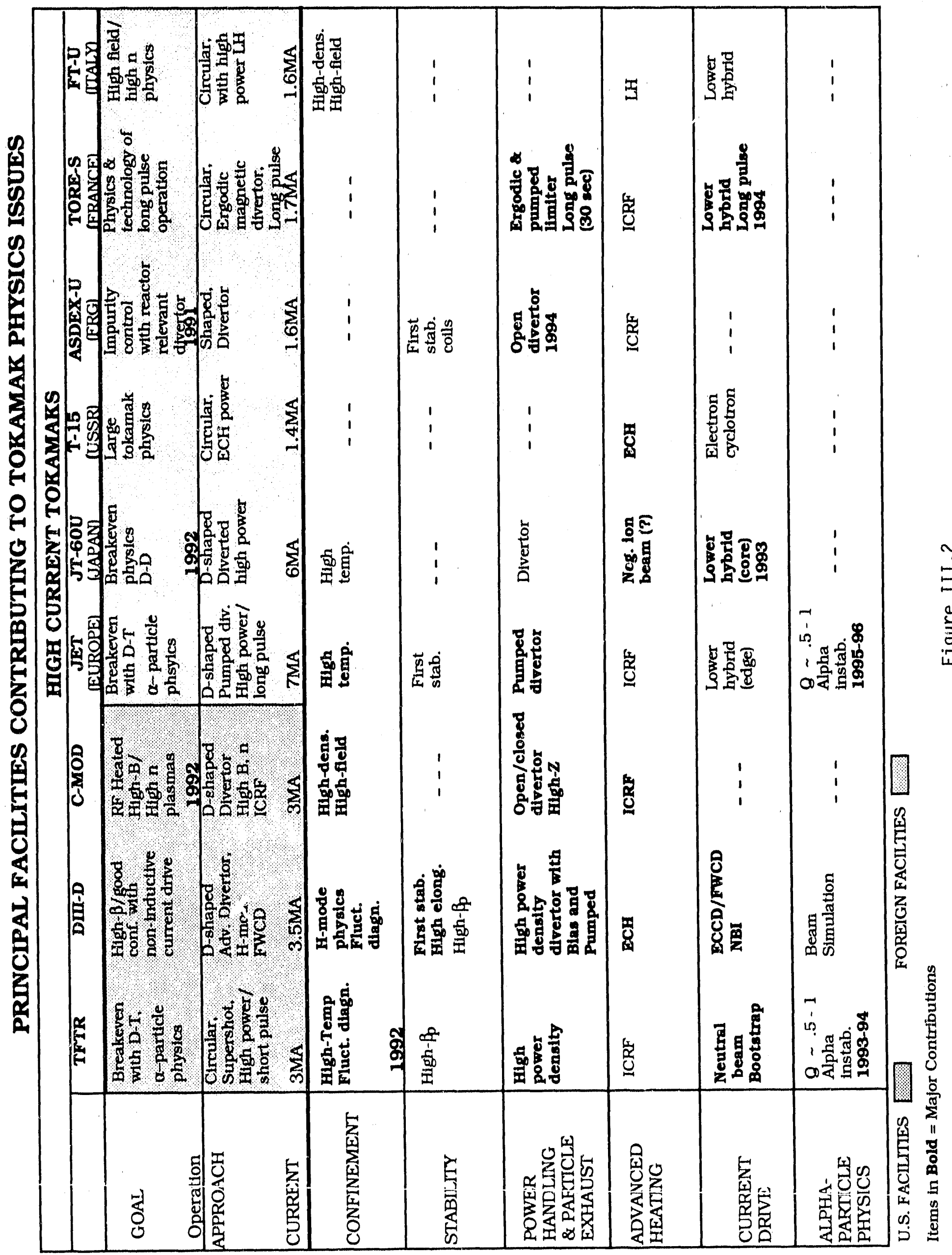



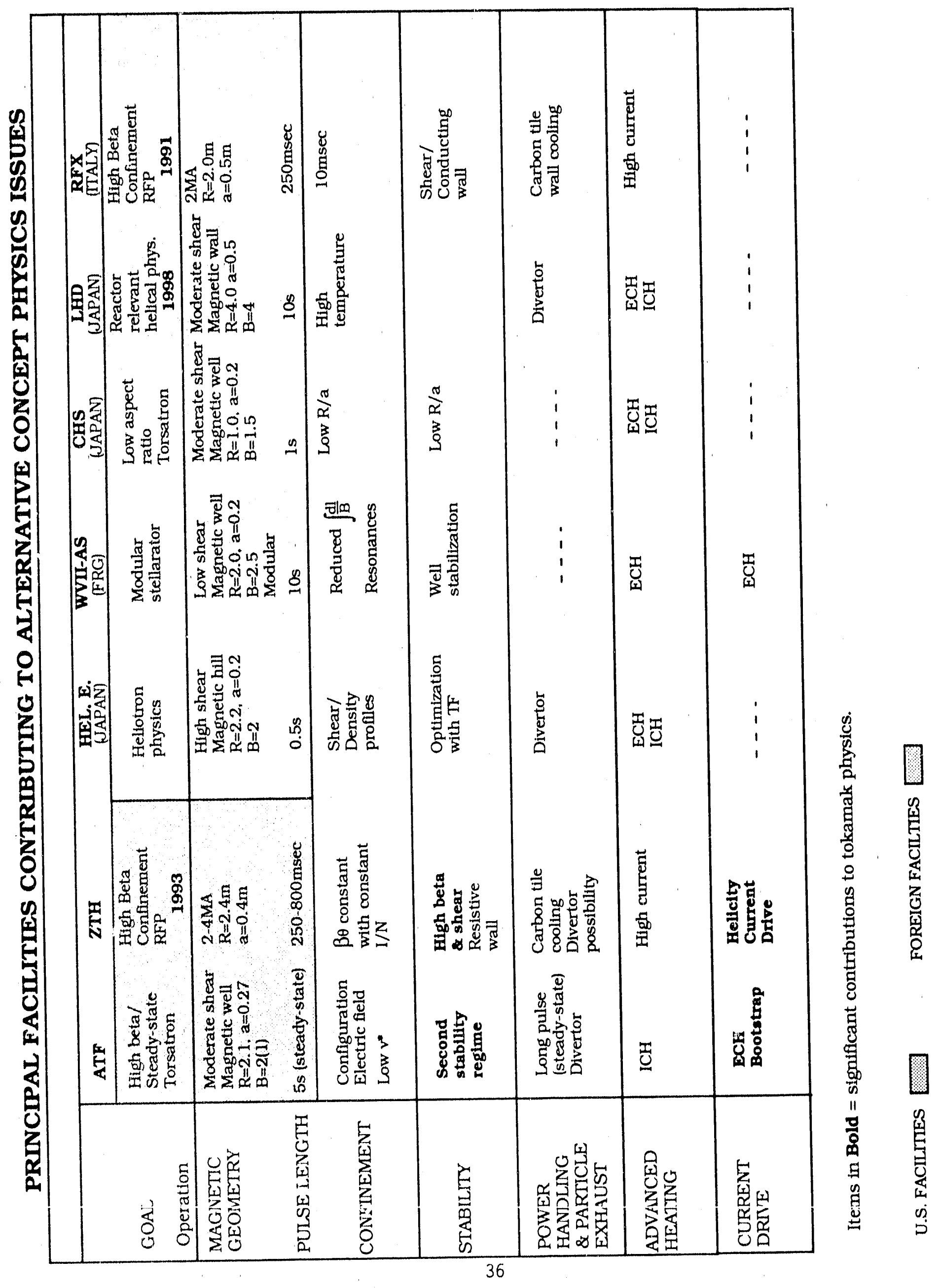


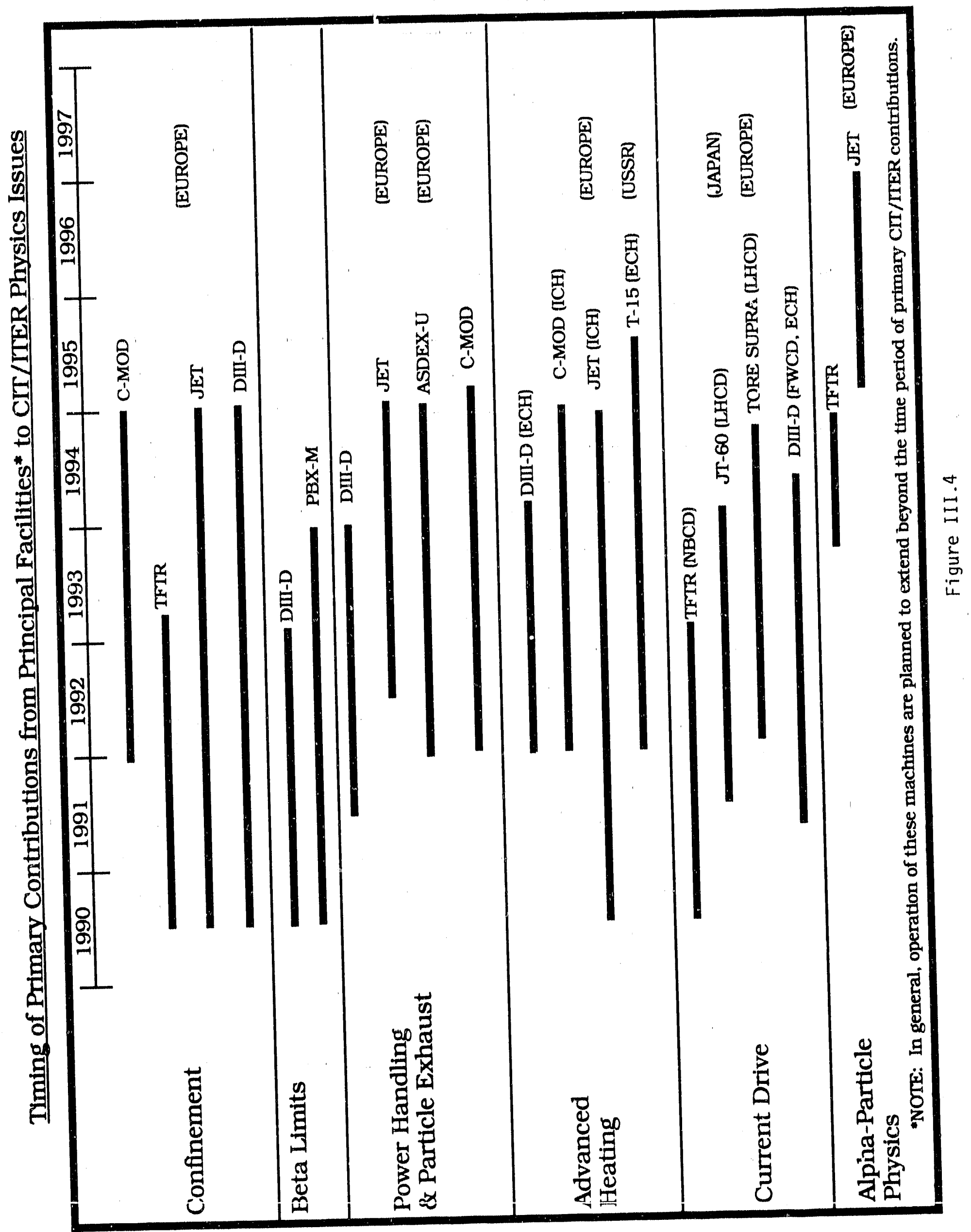






告






\section{CHAPTER IV}

\section{INERTIAL FUSION ENERGY DEVELOPMENT}

\section{A. INTRODUCTION}

The Department of Energy program in inerttal confinement fusion (ICF) is not now an energy program. It is an excellent program within Defense Programs (DP) that is generating information valuable to the nuclear weapons program. It is developing understanding and facilities that will permit the completion of the design and the initiation of construction of a Laboratory Microfusion Facility (LMF), which in turn will have a major national defense role. The program is being reviewed by an NAS/NAE/NRC Committee which issued an interim report on 15 January 1990 and will issue a final report in September 1990.

We accept the NAS judgment that the defense applications of ICF as embodied in the LMF are of great importance to weapons physics and weapons effects studies, and can be realized more certainly and much sooner than the energy goals. These applications would become urgent in the case of nuclear explosive testing limitations. It is thus appropriate that ICF remain primarily a defense program. Nevertheless, the promise of an inertial fusion energy program (IFE) seems to us to be sufficient to begin investment now in a small collateral program covering those areas not required for the DP program, e.g. repetitionrated, efficient drivers and reactor studies. We stress that the energy program makes sense only if the NAS recommended target-physics program is pursued vigorously by $D P$, and care should be taken that adding an explicit energy mission not in any way impede or slow down the ICF progress. Because of the importance and complexity of the ICF program, the Division of Inertial Fusion should regain the status of the Office of Inertial Fusion within DP.

In addition to the different point of contact within DOE, there are four important differences between IFE and MFE:

1. There is a direct defense application for inertial fusion, and the energy program can build on the accomplishments of the defense program. This relation has resulted in classification of part of the program, a complication that we address later.

2. Experiments (Centurion/Halite) using a tiry fraction of the energy from a fission bomb underground have allowed demonstration of excellent performance, putting to rest fundamental questions about basic feasibility of attaining high gain.

3. In all realizations of IFE the driver, the most expensive and extensive element, and the reactor chamber where fusion takes place are separate, which produces savings in cost, improved access, ease of maintenance, and reduced concerns for safety and radiation contamination. 
4. In contrast to MFE, the U.S. Inertial fusion program, despite increasing foreign efforts, clearly remains the world leader with an excellent opportunity to capitalize on that position.

In the inertial confinement approach, a small pellet containing the heavy hydrogen isotopes deuterium (D) and tritium (T) is rapidly compressed to extreme densities (a thousand times liquid density) by very high-power laser or particle beams. Although the dense fuel, heated by the compression, reacts very rapidiy, it is restrained by its own inertia, so that substantial burning (more than $30 \%$ of the fuel) can be achleved before the pellet matter disassembies. A clear understanding of all aspects of pellet compression and burn is obviously necessary. These include, for example, the energy absorption processes (and any hot electron generation mechanisms), instability growth during implosion and any resulting mixing of pellet layers, the conditions for ignition, and the physics of a propagating thermonuclear burn.

For energy applications, there are additional critical problem areas that must be successfully addressed:

1. Pellets must be designed that yield high gain, and can be cheaply produced, efficientiy driven, and stably imploded.

2. Efficient, high-power drivers must be developed that can be operated at useful repetition rates.

3. Reactor chambers must be designed that contain the micro-explosion products and adequately protect the driver.

Although inertial fusion is less mature than magnetic confinement in terms of demonstrated laboratory performance, progress in inertial fusion has been remarkably rapid since its initiation in 1963. Fusion energy yields of about $1 \%$ of the driver pulse energy and compressions of hundreds of times liquid density have been achieved. The long term progress of ICF toward ignition and net gain is shown in Figure IV. 17 on page 54.

\section{B. CURRENT PROGRAM}

The current ICF prograin is supported by DOE Defense Programs with the goal of developing a laboratory capability for examining important weapon physics issues and for developing an advanced above-ground weapon effects simulation above capability. There are four driver aiternatives, two types of targets, and involvement by several DOE Laboratories, other federal 1aboratories, university laboratories, and private industry. The program now has several principal elements:

1. Target Physics. Most of the effort has been on experiments (and on creating the apparatus on which to do them) to enhance the understanding of such high-energy-density phenomena as: a.) the coupling of laser energy to the target, either directly or, more usually, through an intermediary radiant enclosure (hohiraum);

b.) techniques of avoiding premature heating of the fuel so that a high-compression implosion can occur; c.) drive uniformity and 
target preparation to permit high convergence; d.) hydrodynamics of target implosion behavior. These and other issues have been addressed with a vartety of capsule (pellet) destgns. Significant research using planar targets for coupling studies has also been done.

2. Driver development. There has been substantial progress in the development of glass lasers, which have been used for nearly all of the target physics experiments. KrF lasers entered the program later and have not as yet irradiated spherical targets. Light tons (LI), hydrogen and helium, are now being accelerated and focused. Heavy ions (HI) are being explored in a sclence and engineering project supported by Bastc Energy sciences. Except for heavy tons, none of this work has been explicitly oriented toward an energy driver, which must satisfy efficiency, repetition rate, cost, and reliability criteria.

3. Laboratory Microfusion Facility (LMF). This facility, currently in planning, would have a driver energy of 5-10 MJ, and would demonstrate ignition and sufficiently high yield (of the order of $100 \mathrm{MJ})$ to be very useful for defense applications. It would also provide extremely valuable information for the design of fuston energy systems, but it is a large step beyond current experience and is expensive. It now seems prudent to construct an intermediate-size (1-2 MJ) facility to demonstrate ignition and modest gain prior to authorizing a full-scale LMF.

4. Reactor Concepts. At present these are at a preliminary level, and there are inadequately resolved issues that need attention, such as target fabrication costs, final optics or focusing magnet protection, attainable driver efficiencies, and chamber environment.

There is also substantial overseas work on reactor concepts and on drivers, notably at Darmstadt (GSI, heavy ions) and at Osaka and Limeil (glass lasers). Although world-wide there is a great deal of research on target physics and pellet implosions, international cooperation in the target physics area is impeded by U.S. classification and technology transfer policies.

\section{KEY PHYSICS AND TECHNOLOGY ISSUES}

\section{C.1. Target Physics}

The NAS Interim Report recommended that the highest priority in the ICF program be given to studies of target physics, and concluded that this priority would appiy even for an energy motivated program (IFE). We concur with this judgment, noting that the outcome of these studies will determine the minimum size and other characteristics (direct or indirect drive and other choices) of an LMF or Engineering Test Facility (ETF) driver. 
The NAS recommended a two-phase program to investigate target physics: phase 1 should use existing or near term factlittes with the following priority order:

1. Precision Nova, to address hohiraum phystcs and the syimmetry and implosion physics of hydrodynaintcally equivalent targets.

2. Construction of the Omega Upgrade Laser to investigate direct drive and Nike to study smoothing techniques for KrF. It is not yet clear whether indirect drive or direct drive will ultimately prove more useful for defense or energy.

3. Conversion of the Aurora KrF laser into an implosion factlity with which to make comparisons between KrF and glass laser performance on targets. (This NAS recommendation may be modified since Aurora is now thought to be unsuited for implosion physics experiments.)

Following a $3-4$ year study at somewhat increased budget levels in Phase I, a decision could be made on whether to proceed with a 5-10 MJ LMF.

Since the Jamuary 1990 issuance of the NAS interim report there has been an important theoretical advance at LLNL. Numerical simulations have indicated that by going to shorter pulses and higher hohlraum temperatures, already attained on Nova, it should be possible to achieve ignition and modest (5-10) gain implosions with a 1-2 MJ driver. This could be accomplished by an upgrade of the Nova laser in the existing building. This approach would be considerably (2-3 times) less expensive than a 5-10 MJ driver and represents, in our opinion, a more logical next step than a full LMF. Pellet ignition by about the year 2000 is an attractive goal that can probably be attained if construction of the Nova Upgrade starts in FY 1994, as we recommend.

Several modifications to the NAS strategy should accompany a dectsion to proceed with this ignition facility:

1. There would not be time to achieve adequate beam balance on Nova for the fuil hydrodynamically equivalent program to a convergence ratio of 30; a "go, no-go" decision would be based on easier 15-20 fold convergence studies. The adequacy of this data base, especially the cause of deviations from the predictions of onedimensional codes, must be carefully considered. Detalled measurements of yield and density under different conditions would be required to allow extrapolation to ignition.

2. The suggested time for decision would not permit a meaningful competition from KrF or light ion drivers for the next facility. Since Aurora is not suitable for meaningful implosion studies, there could not be a detalled comparison between Aurora and Nova implosion results prior to an ignition facility decision.

We believe that an early decision should be made to proceed with a glass laser ignition facility: Burn should be obtained and the threshold conditions (the "ignition cliff" region) can be fully explored and documented, even if actual 
full ignition were not achleved. Invaluable guidance for the whole DP and energy programs would result: DP could confidentily design and bulld the LMF", and the energy program would have the target physics knowledge it needed to concentrate on effictent repetition-rated drivers, reactors, and target factories.

If the Nova Upgrade is authorized soon, the roles of the KrF and LI progralis should be reconstdered in the context of LMF and energy. The NAS panel has had more opportunity to consider the detalled path among the vartous options; their final conclustons, which should be avallable in September 1990, are not expected to differ substantially from our recommendations above.

\section{C.2 Drtvers}

For energy applications, drivers for inertial fusion must exhlbit good target coupling, be capable of efficient operation at repetition rates of a few hertz, and be reliable and affordable. In many ways lasers are tdeal for trradtating experimental inertial fusion targets. They can generate very short pulses that can be focused onto the target with lenses or mirrors. However, they may be too expensive and tnefficient for energy production. Interest in 1ight-and heavy-ion accelerator-drivers has artsen primarily because the energy absorption processes are well known and the accelerator technologies are believed to be efficient ( - 25\%) and inherently capable of repetitive pulse operation. However, there are important beam transport issues and potentlal problems assoctated with the final focus onto the pellet that must be satisfactorily addressed.

Thie product of driver efficiency and pellet gain should be 10 or more in order that $30 \%$ or less of the developed power is consumed in the driver. Current estimates put indirect drive gains at about 100 and direct drive up to perhaps 300 (higher potential implosion efficiency). Thus a vigorous target physics prograni is essential to determine the required driver efficiency.

Uniess "breakthroughs" in solid-state pumping or advanced target designs prove to be feasible, the inefficiency, expense, and necessary cooling associated with glass lasers eliminate this driver technology from further consideration for energy applications. Of the three remaining candidates, all appear to have favorable target coupling characteristics. The energy deposition process of both light and heavy ions is expected to be classical, or even enhanced due to the additional stopping power of the free electrons. For light fons there is essentialiy no problem of hot electron pre-heat, and the likelthood of such a problem with heavy ions is sma11. For KrF lasers, the wavelength is sufficiently short that hot electron generation is not expected to be a serious issue, although this requires experimental verffication with reactor.like parameters. Thus, the important issues for selecting a driver technology to develop for energy applications are efficient beam generation and transport; ability to focus the beam energy onto a target; ability to operate at repetition rates of a few hertz; affordable development, construction, and operating costs; reliability; and compatibility of the final focusing element with the reactor radiation environment. 


\section{C.3 Heavy Ion Drivers}

Both the 1986 NAS Report and the 1990 NAS Interim Report have suggested that heavy ton accelerators may well be the driver of choice for energy applications: Heavy ion (HI) drivers use the technology of accelerators bullt for expertmental phystcs, which have demonstrated all of the above technical requitrements. The accelerator would be physlcally separated from the reactor chamber by a long beam transport line so that only the final focusing magnets, need to be hardened or shielded from the neutrons.

A heavy ton fuston driver dfffers from accelerators already constructed, in that it must transport very intense bunches of slow (much less than the veloctty of itght) charged particles. The formation, acceleration and transport of such beams, heavily influenced by space charge effects, ratses new issues of beam quality and stability. These issues have been studied experimentally and theoretically in the Heavy Ion Fusion Accelerator Research (HIFAR) program at the Lawrence Berkeley Laboratory (LBL), supported by Basic Energy Sciences (BES) within the DOE Office of Energy Research (OER). The program has successfully resolved the technical issues of multiple-beam acceleration, transport of a heavily space charge loaded beam, and current multiplication in a linear induction accelerator. However, the relatively low currents in HIFAR have not permitted testing of beam-structure interactions or final beam focus characteristics.

The "most logical next step," to adopt the words from a 1986 JASON revtew, is to build the Induction Linac systems Experiments (ILSE) to demonstrate as many of the characteristics of a full sized driver as possible in a scaled-down experiment. ILSE is expected to require four or five years to complete at a total cost, including the base program (currently at $\$ 6 \mathrm{M} /$ year), of about

$\$ 90 \mathrm{M}$; this is the final step of the HIFAR Program. In parallel, enhanced theoretical and experimental efforts are required for an improved understanding of potentially serious longitudinal beam instabllity issues.

It is clear that BES is not an appropriate office for a technology development program, such as HIFAR, which is a relatively large project. If it is to make the logical step to ILSE, the Committee feels the HIFAR program should become the first principal element of a $n a w$ Inertial Fusion Energy (IFE) Program in OFE.

If HIFAR affirms the HI objective, a substantially larger Heavy Ion Driver Demonstration (HI Demo) facllity would need to be constructed as an intermediate technology step between ILSE and a full-scale, multi-megajoule, fusion driver. The HI Demo would not be constructed at $L B L$, but rather should be located where it could ultimately be expanded and upgraded to become the driver for an Engineering Test Facility (ETF). In order to be ready to proceed to this step, if the Nova Upgrade experiments determine the requirements for ignition by about the year 2000, the HI Demo should be constructed toward the end of this decade. It is anticipated to be a $\$ 200-\$ 300$ M project capable of delivering $30-100 \mathrm{~kJ}$ on target. To prepare for the construction of the HI Demo, a relatively small Heavy Ion Driver Technology Program, averaging \$8-10 M per year, should run in parallel with the ILSE project, but starting about one year later. Requirements 
for beam emittance and compatibility of final focusing and beam transport with reactor designs should be carefully studied prtor to HI Demo construction.

As noted earlier, there is a substantlal effort in developing $H I$ accelerator drivers in other nations, notably West Germany, the USSR, Italy, and Japan. West Germany in particular has constructed impressive experimental facllittes in conjunction with the GSI heavy ton physics research center at Darmstadt. A11 of these programs use the more well-developed technology of RF acceleration technology in contrast to the Induction Linac (IL) approach on which the LBL Program is based. The US Program selected IL when HIFAR began in FY 1984. The cholce was partiy because of limited funds, partly because it was known that other nations would follow the RF approach, and partly because of advantages of cost and simplicity of the single-pass IL approach compared with the RF approach, which requires a system of storage rings for stacking and beam manipulation. Nevertheless, US accelerator scientists work in close assoctation with their foreign counterparts, including an on-going sertes of biennial conferences, and would have access to the information needed to choose between the RF and IL approaches when the time comes to construct a next step, such as HI Demo. A comprehensive review of the choice of technology for the accelerator design would be required before proceeding with the HI Demo. It is reasonable to consider the possibility that the HI Demo using the optimal driver technology could be a joint venture with international partners.

\section{ALTERNATIVE ENERGY DRIVERS}

In order to proceed confidently toward an IFE operating plant in 2025 with a robust and restlient program, alternative technologies should be developed for use if the heavy ion approach encounters obstacles. Both of the following programs have important contributions to make to Defense Programs.

\section{D.1 KrF Laser Program}

Lasers have many advantages as potential IFE drivers. Target physics understanding is advanced, their pulse shapes can be optimized for target compression, and they are well matched to the direct-drive approach.

KrF lasers are currently being employed at LANL with the Aurora laser and at NRL with the Nike laser, which is under construction. In order to efficientiy use the relatively long electron beam pulses, by which the lasing medium is excited, both laboratories use a scheme known as "multiplexing" in which the light beams use paths of varying lengths so as to pass sequentially through the lasing medium. Both lasers were designed to optimize their ability to perform interaction experiments, and not for efficiency or repetition rate. They are capable of only single-shot operation at efficiencies of at most a few percent. 
The issues that are importiant for a KrF laser for IFE include espectally:

1. Electrictiy to Light Conversion Efflctency. About $10 \%$ would be requited to offer a reasonable promise for economical power production. This implites a requirement for target gain between 100 and 200.

2. Pulse Repetition Rate. Reasonable reactor pulse repetition rates are between 2 and 10 pulses per second.

3. Rellability and durability. The driver must operate for hundreds of thousands of pulses between mean fallures. Large areas of highoptical-qualtty surfaces would be, needed which must withstand repeated pulses and reactor radiation and particle fluxes on the final focusing elements.

4. Brightness. The entire 1aser 'must operate with excellent optical quality in order that the necessary intensity can be achleved at the target. For indirect drive these requirements are especially severe, because the intensity needed is very high and only a small percentage of the laser power can be allowed to fall outside of the target spot.

We note that the OFE has recently awarded two contracts for industrial teams to evaluate KrF and HI driven IFE systems. These studies should further define issues of cost and performance for KrF lasers for IFE.

We recommend that a KrF system desi!n with beam quality error analyses including al1 the necessary requirements (brightness, effictency, multiplexing, optics area, cost, etc.) be reviewed by an outside, independent panel. This process should require only a few months. If the preliminary results of the OFE evaluations and the conclusions of the system design review show that KrF is an acceptable candidate for an energy driver, we recommend that a four-year $R \& D$ program be designed to investigate the key issues of efficiency, repetition rate, reliability, durability, and brightness for KrF lasers. Such a program should include a test bed which can incorporate all of the key features of an IFE driver, 1.e., correct pulse length, bandwidth, focusability, beam smoothing methods, etc., but need not initialiy be capable of very high energy. It should aim at defining the design of a system that could be scaled first to the $100 \mathrm{~kJ}$ level and finally to the multi-megajoule level needed for IFE. This proposed study should be designed to cost around $\$ 35 \mathrm{M}$ over four years.

\section{D.2 Light Ion Program}

Light ion fusion drivers may be the least expensive option. The most promising concepts are also based on induction linac technology and thus share with HI beneficial efficiency and repetitive pulse characteristics. Moreover, the modular Hermes III technology proposed by SNL for this approach has demonstrated good rellability at reasonable cost and is currently performing well in the nuclear weapons effects simulation program. Thus light ion drivers may actually be closest to meeting the needs of an IFE driver for economic power production. But LI drivers have not yet demonstrated that they can achieve the 
requitred intensity and pulse shape. Furthermore, there rematn issues of generating, transporting, and focusing beanis of adequate quality. To settle these questions in a time reasonable for LI to compete with KrF lasers for a down-selection in FY 1996, the present Sabre expertments at SNL would need to be enhanced.

For example, the Libra LI IFE reactor concept requires the effictent production of l. It ton beams from multiple magnetically-insulated extractor ion diodes, the efftcient and stable propagation of these beams efther ballistically or in plasma channels, and the focusing of these beams onto a pellet. Acceptably low divergence (beam emittance) at each step is a critical issue. We advocate an enhanced effort to resolve these issues satisfactortly by the end of FY 1993. If reasonable progress is being made, a conceptual design review (CDR) for a LI driver for energy applications would be inttiated at that time. Following this two-year effort, intensified work on the design of targets sultable for LI, and continued resolution of the key beam transport issues, a down-selection would be made to a driver technology (etther LI or KrF) based on all avallable information in FY 1996. If $L I$ is the successful competttor, we recommend construction of a $10 \mathrm{MV}, 5 \mathrm{~Hz}, 100 \mathrm{~kJ}$ module, capable of delivering $50 \mathrm{~kJ}$ of ion energy to a target over a $2 \mathrm{~m}$ to $4 \mathrm{~m}$ beam transport path. It is estimated that this module would be constructed by the end of FY 1998 and avallable for beam generation, transport, and focusing demonstrations. Successful completion of these efforts by the end of FY 2000 would then permit another down-selection, in this case between LI and HI, as the IFE driver of chotce.

Additional funding for the initial Sabre experiments (above that provided by $D P)$ is expected to require $\$ 5 \mathrm{M}$ per year, increasing to $-\$ 12 \mathrm{M}$ per year in FY 1995. The conceptual design activities are estimated to require a total of $\$ 4 M$ over a two year time period. Construction costs for the $100 \mathrm{~kJ}$ repetitively operated module are expected to be in the neighborhood of $\$ 100 \mathrm{M}$.

\section{E. COMPLEMENTARY ENERGY PROGRAM}

In addition to driver development, there are several other program elements that should be a part of the energy program.

\section{E.1 Fuston Materials Development}

This is an area that has been largely cut from the MFE Program. There is great simllarity in the needs for materials for reactor chambers, blarikets, etc., between MFE and IFE. An accelerator to generate $14 \mathrm{MeV}$ neutrons is needed for radiation effects studies for both programs. We assume this will be provided under the MFE program. The IFE materials program might reasonably be about $\$ 5$ $M$ per year to develop materials and materials understanding specifically for IFE reactors.

\section{E.2 Reactor Design Studies}

In recent years, a small reactor R\&D Program has been funded from the ICF Division. Several rudimentary designs for IFE reactors have resulted from this work and constitute the basis for IFE cost studies suggesting that the cost of 
electricity from IFE could be competitive with other energy sources. Various advanced breedisig blankets have been studied for MFE and were documented in the ESECOM study; one of these, a lithium-beryllium-fluoride compound, looks particularly attractive as a blanket for IFE. Several design variations have a) so resulted from studies in Japan and from the collaboralions between the University of Wisconsin and West Germany. These designs take different approaches to protecting the first wall and generating the needed tritium. The OFE has recently awarded two $\$ 2 M$ contracts to industrial teams to evaluate different reactor and driver systems for IFE. This is rotentially a very high leverage area of research since design considerations might well narrow down driver options more cheaply than could large driver demos. Funding at the $\$ 5 \mathrm{M}$ per year level for studies and limited experiments should be initiated as soon as possible. Such subjects as "wet-wall" protection, beam transport requirements, and the survivability of the "last optic" or "final magnetic focusing" are crucial issues.

\section{E.3 Target Factory}

Studies should be undertaken of the techniques for production of target pellets, including cryogenic pellets. We suggest little more actual fabrication than the work thac is being done for target physics until reactor and driver selections are bet,ter defined, but the requirements for a target factory should be kept in mind. and an appreciable program (\$3 M to \$5 M per year) should be started well befure 2000. An answer is needed to the question: Can targets (especially indirect or advanced ones) be mass produced and positioned as cheaply as required? At least a plausible solution to this issue should be provided before very expensive energy drivers (e.g., a HI Demo) are built.

\section{E.4 Advanced Fuels}

Advanced fuels, including helium-3, have been proposed to reduce the need for tritium and also to reduce the neutron flux, but unrealistically large drivers may be required for them. We do not now suggest additional funding; funding would become substantial only if serious system prospects become apparent.

\section{F. DEFENSE AND ENERGY PROGRAM STRATEGY}

Earlier in this chapter recommendations have been made for additions to the current Defense Programs funded activities, in order to initiate an energy program. The IFE development program is summarized in Figure I.2 on page 10. The defense program and applications would not be sacrificed, and in fact would be enhanced and accomplished sooner. For example, ion drivers could turn out to be useful for the LMF. But the big change is that the program would provide an attractive option for a major source of electrical power by the middle of the next century and would capitalize on the technological lead that the $U$. S. now enjoys in ICF.

Our recommendation is solidly for the full program of the next four sections, but we have been charged to recommend priorities in case the full program cannot be established. We do so in four blocks. Even the first of these which would be done with minimum funding, begins with annual funding greater than the 
current leve1. The additions in this first block are primarily those recommended in the NAS study, to make the DP effort more effective and efficient, and secondarily those required for a very modest start on an energy program.

The second block adds the logical step for both defense and energy; without this, the defense program will slow and the energy program will not get an effective start.

The third and fourth blocks add the elements required to proceed with an energy program. The budget increment for block three is just half of that for block four.

Funding to implement the IFE budget should not come from existing DP or MFE budgets.

A11 four elements are needed to follow the energy schedule of Figure 1.2 on page 10. The energy part of the program could be delayed, of course, but with the usual consequences of costing more in total. If, because of a comprehensive Test Ban or other restrictions at the Nevada test site, the LMF is needed more urgently, the Defense part could be advanced somewhat, with increased annual funding but little change in the total.

Down-selection of alternative drivers has been incorporated in the plan. Additional savings in both defense and energy funds may result from the NAS final recommendations. We believe our program and recommendations will be consistent with the NAS study, but clearly the DOE will wish to compare the two reports, especially with respect to the immediate question of the Nova Upgrade.

\section{F.1 First Priority, Defense and Energy}

The first priority for both defense and energy is to continue the current DP target physics program with the additions recommended by the NAS panel in January 1990. The principal additions in FY 1991 are to modify Nova to Precision Nova, to begin construction of the Omega Upgrade, and to accelerate construction of Nike. If nothing is dropped, the FY 1991 figure would be approximately: LLNL (Precision Nova), \$95 M; LANL (Aurora), \$37 M; SNL (PBFAII), \$31 M; UR (Omega), \$30 M; NRL (Nike), \$6 M; target support and other, $\$ 12 \mathrm{M}$. These total about $\$ 44 \mathrm{M}$ more than the budget submission of

FY 1991. Congressional action is stili unknown at this time. As we have noted above, we regard Precision Nova, the Omega Upgrade, and the completion of Nike as the highest priority items. In the absence of full funding, cuts may have to be made by changes in the KrF, LI, and target support programs.

Even in this low-funding scenario, we recommend beginning the ILSE program and continuing the OFE reactor studies program.

This first priority program would continue through at least the next few years, and even without cutting any programs would cost annually only $\$ 20 \mathrm{M}$ to $\$ 40$ in more than DP planning figures, including construction and other capital costs. 


\section{F.2 Second Priority, Defense and Energy}

The Nova upgrade to an energy of 1-2 Mu should be authorized as soon as possible after meeting the milestones set by the NAS review, with preparations for a CDR leading to a Title I in FY 1992 and a construction start in FY 1994. First experiments in FY 1997 will underpin both an LMF (defense) and an ETF (energy). Very few additional funds would be spent in FY 1991, about $\$ 9 M$ in FY 1992, and then the major construction funds in the subsequent years totaling approximately $\$ 320 \mathrm{M}$ (in then-year dollars). Authorization for the upgrade should be 1 imited to a construction figure of perhaps $\$ 380 \mathrm{M}$.

The schedule above is set to produce information and experience in a timely fashion for LMF and ETF. While design and cost validation are proceeding, experiments on Precision Nova, Omega, and possibly Aurora will give data to resolve any remaining physics issues before actual construction begins. After initiation of construction, further Nova, Omega Upgrade, and Nike results will permit refinements and improvements and help shape the ignition regime experimental program on the Nova Upgrade.

This activity is the logical next step and is not included in the first priority only because it is a substantial expenditure and need not start immediately.

\section{F.3 Third Priority, Energy}

In order to start an energy program, work on drivers is essential. Figure IV.2 on page 55 shows a schedule which develops HI plus two alternative ( $K r F$ and LI) drivers. For this priority, only one-half of the annual increments for a full energy program are assigned, and only the HI driver research can be supported.

Table I gives the funding profile for the next few years for this priority. Note that $\$ 6 \mathrm{M}$ per year is already being spent (in BES of OER) and is included in the first line. The increment in FY 1992 of this 7 ine to create an energy program is thus $\$ 4 \mathrm{M}$.






\section{F.4 Fourth Priority, Energy}

Only here do we get the full program for the next 5-6 years as described in this Chapter and outlined in Figure I.2 on page 10. Funding for these years is scheduled in Table II. In FY 1996 and thereafter the program would approximately double with the construction of HI Demo and an alternative.



\section{G. ORGANIZATIONAL ISSUES}

We have already mentioned our first and most urgent organizational recommendation: The present Division of Inertial Fusion within Defense Programs should become, as it previously was, the Office of Inertial Fusion. This is the appropriate level in DOE since the present structure is inadequate to maintain the required status of ICF in DP.

The second, also immediate, recommendation is to provide an appropriate "home" for heavy ion research and development. HIFAR is an organizational misfit in Basic Energy Sciences. There is no possibility of supporting a multi-milliondollar, goal-and-schedule-oriented development program with in BES. We recommend that a new Division of Inertial Fusion Energy be established within the Office of Fusion Energy and that heavy ion driver development be part of its program.

The new Division of Inertial Fusion Energy with OFE should also manage and direct the other items in Table II. This will permit a systematic approach to energy and lead to a more integrated energy program. Although there will be an organizational interface created at a National Laboratory e.g., in the KrF program at LANL, we believe that intimate mutual reinforcement of the two parts can be provided at the Laboratory level. 
There should be a standing outside review committee established, as recommended in the 1986 NRC ("Happer") report, advising both this new Division and the office of Inertial Fusion in DP.

\section{H. CLASSIFICATION ISSUES}

Some parts of indirect drive research are now classified. Although those not having access to classified material tend to over-estimate its extent, there are real drawbacks to the present situation. These will become much more onerous if an IFE energy program is undertaken. The disadvantages include:

1. Restricting the size and variety of the community that can contribute to, criticize, and evaluate the target physics program. This is already seriously limiting progress and will become more damaging in a broader energy program.

2. Restricting international collaboration and wide participation by industry, especially serious in a high-technology program.

3. Creating a vulnerability to public misunderstanding.

4. Cost of guards, secure facilities, handling documentation, etc. This is by far the smallest cost.

The third item, although intangible, is probably the most important penalty of unnecessary classification. By about 2025, if an operating power reactor is built, public understanding of every part of it, including targets and the target factory and the technical specifications, will be absolutely necessary to convince the public of its safety. We cannot predict accurately how long before this date declassification would be necessary to avoid irreversible commitment of protest organizations, media misunderstanding, and public concern. But declassification surely must precede construction by many years. On the other hand, with sensitive management and information flow, declassification does not seem to be required immediately upon announcing an IFE energy program.

Thus the question: Will the current slow reduction in classification of ICF experiments and pellets be fast enough to provide a feeling of confidence in the public mind? We are apprehensive about this and we know that classification reviews take a long time. We therefore recommend that the Secretary of Energy form a high-level panel of independent, outside experts to review present ICF classification guidelines and recommend a schedule of future target physics declassifications that would be required for energy applications. This review should include an evaluation of future international developments that might result in the publication of presently classified materials.

In addition to dealing with public perception before it becomes an irreversible problem, declassification would have the immediate benefit of fostering the international collaborations that have great potential benefits for the program.

In addition to classification, there are other regulations and practices of DOE and the Department of state that go well beyond classification to make 
international visits and exchanges difficult to arrange and less productive. An atmosphere like that existing in MFE needs to be created, in wintch vigorous national programs are preserved within an international community. In this environment, individual scientists easily establish cordial, and productive relations with their counterparts from other nations.

With progress toward this goal in mind, we make a specific recommendation, that the Secretary of Energy should initiate discussions with the State Department (Ambassador Kennedy, et a1.) regarding possible international collaboration, consistent with present classification guidelines, on IFE driver development for energy applications. Present policy permits some international cooperation where the benefits clearly outweigh any potential risks. Possible initial areas of collaboration are heavy ion accelerators, laser drivers, materials, reactor concepts, and environmental impacts.

\section{CONCLUDING REMARKS}

We believe that the program we have outlined for ICF and IFE is a sound and efficient program that is timely for several reasons.

First, the current rethinking of our defense posture is likely to lead to a reduction of underground testing with lower permitted yields. Although ICF is not a substitute for underground testing, its AGEX and simulation capabilities should make it very valuable in the new situation, and the energy program can make use of, and build upon, this capability.

Second, a large effort is now underway to redirect the aerospace industry and DOD and DOE Laboratories into efforts useful for the future well being of the Nation in a world less threatened by major conflict. Surely a safe and inexhaustible energy supply must be high on the long-range agenda, and it is difficult to imagine a critical high technology venture that is more suited than IFE to the talents in these national assets. Fusion is the most promising and highest technology "plowshare" available.

Finally, we believe that the recent successes of Centurion-Halite and laboratory experiments, with associated theoretical developments, provide for inertial fusion a special window of opportunity in the near future. 










APPENDIX 1

CHARGE TO THE COMMITTEE 
Dr. H. Guyford Stever

1528 33rd Street, N.W.

Washington, D.C. 20007

Dear Dr. Stever:

The Department of Energy is supporting research and development in two major areas of fusion research, magnetic confinement and inertial confinement. Last. year, a new fusion policy for the Department was proposed by the former Director of Energy Research, Dr. Robert 0 . Hunter. A key feature of this proposed policy was competition between the two major confinement approaches.

The Fusion Policy Advisory Committee (FPAC) has been formed to take a broad look at what the Department's fusion policy should be. While the enclosed charter establishing FPAC discusses the Committee's review in relation to a specific policy proposal, I wish to emphasize that my primary interest is in having the committee provide its best judgment on the optimal way to structure the overall U.S. fusion program. For example, FPAC should feel free to address the question of whether or not there should be a competition between magnetic and inertial confinement.

Developing fusion energy has proven to be an extremely difficult undertaking. Substantial progress has been made in both the magnetic and inertial confinement programs but neither has progressed to the point where a practical fusion reactor can be built. I look to your Committee to help the Nation establish a wise, practical, and enduring policy for fusion, one that will enable fusion energy to be a valuable energy source in the next century.

The "Magnetic Fusion Energy Engineering Act of 1980, "Public Law 96-386, established a Technical Panel on Magnetic Fusion of the Energy Research Advisory Board (ERAB) to conduct a triennial review of the conduct of the national magnetic fusion energy program. Since such a review is essentially part of the charter of FPAC, I would like the Committee, in conjunction with its work, to perform this review. I will ask the ERAB Chairman to appoint members of your Committee to serve as the Technical Panel on Magnetic Fusion.

Thank you for your willingness to chair this important activity for the Department of Energy. I hope to receive an Interim Report from the Committee in July and your Final Report by the end of September.

Enclosure

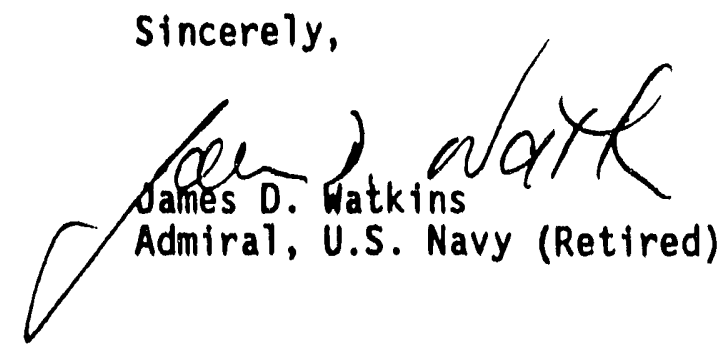




\section{The Secretary of Energy}

$W$. shington, DC 20586

May 14, 1990

Mr. John Landts

Chatrman

Energy Research Advisory Board

1000 Independence Avenue, SW

Washington, D.C. 20585

Dear Mr. Landis:

The Magnetic Fusion Energy Engineering Act of 1980 (Public Law No. 96-386) estabilshed a broad basis for the future development of magnetic fuston energy. The Act provides for a five-year comprehensive program management plan and a series of steps to lead to determining the engineering basis for fusion development.

The Act also requires that an overall review of the conduct of the magnetic fusion program be undertaken by a Technical Panel of the Energy Research Advisory Board (ERAB) on at least a triennial basis. In particular, the Act specifies that the review shall consider, among others, the following topics:

- the five-year program management plan,

- future facilities needed to meet the goals of the Act,

- the adequacy of participation by universities and industry,

- the adequacy of international cooperation and any problems associated therewith, and

- instituticnal, environmental and economic factors limiting, or prospectively limiting, efforts to achieve commercial application of inagnetic fuston energy systems.

It is now appropriate to activate the Technical Panel of ERAB to perform the triennial review. As you are aware, the Department has formed the Fusion Policy Advisory Committee (FPAC) to take a broad look at what the Department's fusion policy should be. Since the ERAB review is in essence part of the charter of FPAC, I would like you to appoint the members of FPAC to serve as the Technical Panel on Magnetic Fusion so that FPAC can carry out the mandated review. Furthermore, the FPAC final report should serve as the Technical Panel report. 


\section{DEPARTMENT OF ENERGY}

CHARTER

FUSION POLICY ADVISORY COMITTEE

\section{Committee's Offictel Desianation:}

Fusion Polfcy Advisory Comittee

2. Comitten's Objectives. Scope of Activitios and Duties:

The Fusion Pollcy Advisory Comittee w111, on a one-tine basis, review the new draft fusion pollcy covering both magnetic and inertial confinement, and provide its recommendation for a fusion policy for the Department of Energy. The review will focus on the technical basis for, and the likely effectiveness of, the policy proposed. The find written report will provide advice on how to structure the fusion programs -. elther by the proposed polfcy, some spectflc modifications to it, or some totally different approach. The pollcy recommended by this Comittee should include goals and objectives of the programs, a coherant strategy, an outline of the development path, identification of major decision points, determination of the role of international collaboration, and recommendations on funding levels. Spectfic questions that should be addressed include, but are not ilmited to, the following:

1. How should the competition between magnetfc confinement fusion and inertial confinement fusion be structured to assure the successful development of commercial fusion energy?

2. What should be the balance in these programs between an emphasis on burning plasma devices and broadly based physics and technology R\&D?

3. At what point in the program should major burning plasma experiments be undertaken, and when will the level of science and technology be adequate to build these devices with confidence?

4. How should the international emphasis on proceeding with an International Experimental Thermonuclear Reactor (ITER) be accommodated in the new policy?

5. How should driver development in the inertial program be carried out in the new poifcy? Is the approach compatibie with the military objectives of inertial fusion research?

6. What is the appropriate funding for the fusion prograns? Can burning plasma devices and international collaboration be accomplished within current total budget levels? W111 tho proposed transfer of $\$ 50$ million from wagnetic to inertial fusion establish an effective competition between the programs? 
3. Time Perfod Necessary for the Comnittee to Carry Out its Purpose:

The Committee is expected to be in existence for ten wonths.

4. Offictal to Whom This Committee Reports:

The Secretary of Energy, through the Director, Office of Energy Research.

5. Agency Respanstble for Providing Hecessary Support for This Committee:

Department of Energy. Within the Department of Energy, the primary support shall be furnished by the Science and Technology Affairs Staff, Office of Energy Research.

6. Description of Duties for Which Committee is Responstble:

The duties of the comntttee are solely advisory and are stated in Paragraph 2 above.

7. Estimated Annual Operating Costs in Dollars and Man-Years:

$\$ 300,000$ and two man-years.

8. Estimated Number and Frequency of Committee Meetings:

The Committee and any subcomittees established will meet approximately eight times during the year and at such other times as may be requested by the Director of Energy Research.

9. Committee's Termination Date (if less than two years from the date of establishment or renewall:

The Committee will terminate on September 30, 1990.

10. Subcomlt ttees:

Subcamittees may $b B$ established as approprlate, to facilltate the functioning of the committee. The objectives of the subcomnittees are to make recommendations to the parent committee with respect to the Cormittee's activities as cited in Paragraph 2 above.

11. Members:

(a) Committee members shall be appointed by the Secretary of Energy.

(b) Approximate number of committee members: 15

(c) Committee members will be appointed to a term coinclding with the existence of the committee. 
12. Chairpersen:

The Chairperson shall be appointed by the Secretary of Enaray.

This Charter for the advisory comittee named above is hereby approved:

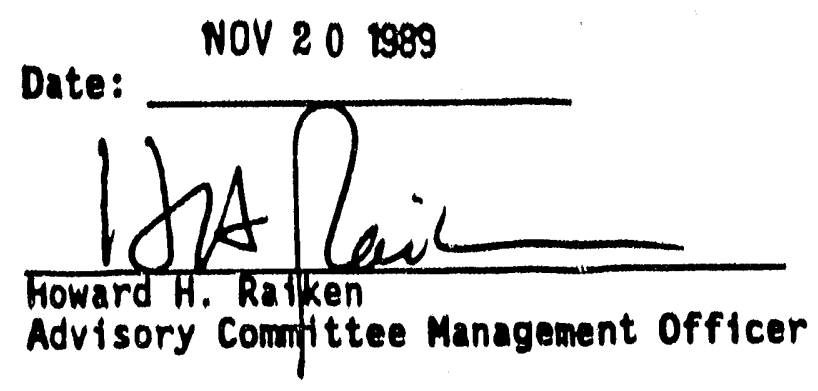

Date Filed: NOV 201989 


\section{APPENDIX 2 SECRETARY'S LETTER AND COMMITTEE'S RESPONSES TO QUESTIONS}


Dr. H. Guyford Stever

Chairman

Fusion Policy Advisory Committee

1528 33rd Street, N.W.

Washington, D.C. 20007

Dear Dr. Stever:

Thank you for your letter of July 23, 1990, transmitting the interim report of the Fusion Policy Advisory Committee. I appreciate the hard work and dedication of the committee in conducting a comprehensive review of the fusion program and in attempting to lay out a course for its future. The cohesion of the committee, which reflects a diversity of viewpoints from within and outside the fusion community, is a testament to your able leadership.

The committee's interim report is a good start towards a comprehensive policy and program for turning nuclear fusion into an energy supply option for the Nation. It articulates a need for a sea-change in the Nation's approach to fusion, from a research program on the physics underlying fusion to an energy development program. It lays out a series of programmatic steps that would be entalled in the optimal approach to fusion energy in which the United States exercises world leadership.

I am concerned, though, that the report, in laying out the ideal path for developing fusion energy, may raise expectations in the scientific community that such a path will be relatively simple to implement in the current budgetary environment. Given the severe constraints that all research and development funding is facing this year, this is not likely to be the case.

To assist me in making hard choices about the fusion program over the next few years, and in defending those choices to other participants in the budget process, I would like the committee to address the following questions.

- Can fusion be made an economically competitive source of energy in the future? A decision to treat fusion as an energy development program must be supported by an analysis that fusion is likely to be competitive with other potential energy supply options. The interim report mentions that, by the middle of the next century, other U.S. energy supply options may be seriously constrained by environmental considerations or public acceptability. In this context, fusion energy seems imperative, somewhat irrespective of 
costs. Should supply options such as advanced fission reactors be viable in the middle of the noxt century, though, would fusion power plants be likely to be competitive with them?

- The interim report spells out a leadership role for the United States in the comerclalization of fusion energy. Does this need to be the case for every step in the process? For wirich steps is it critical that the United States exercise world leadership?

- How can the United States derive maximum value from international collaboration in fusion research and development? I believe that the U.S. plan for fusion has to address the potential contribution to our program of each international facility for fusion research and development that is in use or is firmly committed to.

- For fusion energy to become a viable energy supply option, fusion energy development must eventually attract substantial investment from U.S. industry. Which milestones in the development path laid out by the committee are most critical for industrial involvement? Which have the greatest potential for spin-off to non-fusion technologies?

I believe that the committee, as it moves from its interim report to its final report, must make a critical assessment of each milestone on its identified paths to fusion energy. What is the relative importance of U.S. Teadership on each milestone? How much would be lost if a given milestone could not be accommodated within the U.S. program? Without such a critical approach, I am afraid that the final report of the committee might not command the credibility necessary for it to lead to the creation of a truly effective fusion energy program.

I realize that I am asking the committee to undertake tasks that will be difficult and, perhaps, contentious. Yet, I am depending on committee members to give me their best judgment on these tough questions, so that I can make hard choices in an enlightened manner. I am most griteful for the diligent and patient work of the committee --it has indeed made substantial progress towards defining a fusion energy program that can attract broad and sustained Federal support. I look forward to your continued dedication on this important task for the Nation.

Sincerely,

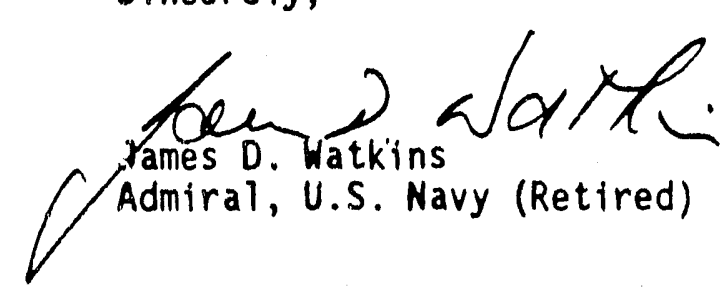




\section{RESPONSES TO QUESTIONS FROM THE SECRETARY OF ENERGY}

\section{Can fusion be made an economically-competitive source of energy in the future? Should supply options such as advanced fission reactors be viable in the middle of the next century, would fusion power plants likely be competitive with them?}

Numerous studies indicate that electricity generated by fusion power plants has the potential to be competitive with that generated by fission or coal plants. Without delays or other constraints, the cost of electricity from fission or coal systems would probably be lower than that from fusion systems, as projected today. The competitiveness of fusion energy arises primarily from its very significant safety and waste-disposal advantages, even compared with advanced fission reactors. Because these factors are likely to dominate politically and economically, fusion energy may be competitive with fission energy despite the higher cost of fusion in other respects. Until public acceptability and its consequences are clear, however, it will not be possible to make a valid economic comparison of fission and fusion. (Common Issues: Economic Assessment of Fusion Power Systems)

The goals established for the U.S. fusion energy program are consonant with this rationale. A programmatic priority must be the characierization and validation of fusion power's safety and environmental promise. This combined with the establishment of a domestic technology base that validates the electric power promise will position the U.S. for realization of the advantages of fusion energy for a long period. (Common Issues: The Current Situation and Expected Future)

2. For which steps is it critical that the United States exercise world leadership in the commercialization of fusion energy? Is it critical for every step?

It is important that the U.S. be competitive in the commercial step. The best way for the U.S. to be competitive in that step is to be a strong participant in all steps leading to commercialization. (Common Issues: Industry Roles in Fusion Energy Developrient)

3. How can the United States derive maximum value from international collaboration in fusion research and development?

Value from international collaboration is obtained from the synergistic effects of sharing knowledge and trained personnel, reduction of financial burdens, spread of risks across a broad base, optimization of the uses of special facilities and capabilities, increased opportunity for spin-offs and the application of technical advances to related fields, and creation of a certain amount of human and political harmony. The current U.S. MFE program involves extensive collaboration in virtually all of its activities. To maximize this interaction, it is imperative that the strengths of the U.S. program be increased. The cooperation in the IFE areas is growing and should be encouraged appropriately. (Common Issues: International Collaboration). 


\section{APPENDIX 3}

\section{MEETINGS AND SITE VISITS}




\section{FUSION POLICY ADVISORY COMMITTEE}

\section{MEETINGS AND SITE VISITS}

March 22-23

March 22, 1990

March 23, 1990

Apri1 2-4, 1990

Apri1 2, 1990

April 3, 1990

Aprit 16-17, 1990

Apri1 17-18, 1990

May 2, 1990

May 3, 1990

May 4, 1990

May 9, 1990

May 10,1990

May 11,1990

May 12,1990

May 14, 1990

May 15,1990

May $24-25,1990$

May $24-25,1990$

June 28-29, 1990

JuTy 23,1990

JuTy 25,1990

JuTy 26-27, 1990
FPAC Policy and Program Consideration Meeting, Washington, D.C.

Evening Subcommittee Organization Meeting

Afternoon Subcommittee Meetings as scheduled by Subgroups

FPAC Policy and Program Considerations Meeting, Germantown, MD

Evening Subcommittee Meeting

Afternoon ICF Subcommittee Meeting

MFE Subcommittee Meeting at PPPL (Princeton/MIT)

MFE Subcommittee Meeting at ORNL (ORNL/ITER)

MFE Subcommittee Meeting at LANL

MFE Subcommittee Meeting at GA (GA/LLNL/UFA)

MFE Subcommittee Report Preparation

IFE Subcommittee Meeting at LLNL.

IFE Subcommittee Meeting at LBL

IFE Subcommittee Meeting at LANL

IFE Subcommittee Meeting at SNL-Albuquerque

IFE Subcommittee Meeting at Rochester

IFE Subcommittee Report Preparation (Rochester)

FPAC Coherent Strategy Considerations Meeting, Washington, D.C.

Generic Issues Subcommittee Meeting

FPAC Interim Report Review, Washington, D.C.

Interim Report Transmitted

IFE Subcommittee Meeting

FPAC Development Path Priorities Meeting, Washington, U.C. 


\section{FUSION POLICY ADVISORY COMMITTEE}

\section{MEETINGS AND SITE VISITS (CONTINUED)}

Ju1y 26, 1990

August 10, 1990

August 21, 1990

August $27-28,1990$
Subcommittee Meetings

MFE Subcommittee Meeting, San Francisco, CA

Subcommittee Chairmen Meeting, Chicago, IL

FPAC Report Review Meeting, Washington, D.C. 


\section{APPENDIX 4}

\section{PREVIOUS REVIEWS}




\section{PREVIOUS REVIEWS}

Koonin, S.E. (Chair). 1990. Interim Report on Inertial Confinement Fusion. Commission on Physical Sciences, Mathematics, and Resources, National Academy of Sctences, Washington, D.C.

White, I.L. (Chair). 1989. Pacing the U.S. Magnetic Fusion Program. Committee on Magnetic Fusion in Energy Policy, National Research Council, Washington, D.C.

Gibbons, J.H. 1987. Star Power. OTA-E-338, Congress of the United States, Office of Technology Assessment, Washington, D.C.

Gavin, Jr., J.G. (Chair). 1986. Report of the Technical Panel on Magnetic Fusion of The Energy Research Advisory Board. DOE/S-0035, Technical Panel on Magnetic Fusion, Energy Research Advisory Board, Washington, D.C.

Hammer, D., et. a1. 1986. Heavy Ion Fusion III. JSR-86-302, Mitre Corporation, Mclean, VA. (JASON Review).

Happer Jr., W. (Chair). 1986. Review of the Department of Energy's Inertia 7 Confinement Fusion Program. Committee for a Review of the Department: of Energy's Inertial Confinement Fusion Program, National Academy of Sciences, Washington, D.C.

Roddis, L.H. (Chair). 1984. Magnetic Fusion Energy R\&D. DOE/S-0026, Magnetic Fusion Energy Panel, Energy Research Advisory Board, Washington, D.C.

Woodson, H.H. (Chair). 1982. Future Engineering Needs of Magnetic Fusion. Committee on Magnetic Fusion, National Academy of Sciences, Washington, D.C.

Buchsbaum, S.J. (Chair). 1980. Report on Magnetic Fusion Program. Fusion Review Pane1, Energy Research Advisory Board, Washington, D.C.

Foster, Jr., J.S. (Chair). 1978. Final Report of the Ad Hoc Experts Group on Fusion. DOE/ER-0008, Fusion Review Committee, U.S. Department of Energy, Energy Research, Washington, D.C. 
APPENDIX 5

GLOSSARY 


\section{5-1 ACRONYMS AND SYMBOLS}

AGEX

ALCATOR C

ARIES

ASDEX

ASDEX-U

ATC

ATF'

BES

C-MOD

CDR

CIT

CPRF

D-D

D-Heltum-3

D-T

DEMO

DIII or DIII-D

DOD

DOE

DP

EC

ECH

ECU

EDA laboratory experimental faclitties for simulation of nuclear weapons effects

tokamak with relatively high current and particle densittes (MIT)

tokamak fuston power concept

Axtsymmetric Diverter Expertiment, closed diverter (Garching, Germany)

upgrade of ASDEX, open diverter (Garching, Germany)

adtabattc toroldal compressor, an early tokamak (PPPL)

Advanced Toroldal Factlity, a large stellarator (ORNL)

Bastc Energy Sciences, DOE-Office of Energy Research

upgrade of Alcator-C factlity (MIT)

conceptual design report

compact ignttion tokamak

Confinement Physics Research Factlity (LANL)

fusion fuel involving only deuterium

fuston fuel involving deutertum and helium-3

fuston fuel involving deuterlum and tritium

demonstration reactor

Doublet III, Tokamak (GA)

U.S. Department of Defense

U.S. Department of Energy

Defense Programs (DOE)

European Community

electron cyclotron heating

European Currency Untt:

engineering design activity 
ERAB

ESECOM

ETF

ETR

FEL

FPAC

FT.U

FWCD

GA

GSI

HERMES III

HI Demo

HI

HIFAR

$H$-mode

ICF

ICH

ICRF

IFE

II.

II.SE

ISX-B

ITER
Energy Research Advisory Board

Sentor Commtttee on Environmental, Safety and Economics; organtzed by DOE in 1985 to provide an assessment of magnetic fuston energy's prospects

energy test factlitiy

engineering test reactor, device in U.S. fuston strategy

free electron laser

Fuston Poltcy Advisory Committee

Frascatf Tokamak Upgrade; a high density, high current tokamak (Frascatt, Italy)

fast-wave current drive

General Atomics (San Diego, CA)

Gesel1schaft fur Schwertonenforschung (Darmstadt, Germany)

an accelerator at SNLA but1t and operated for simulating nuclear weapons effects.

heavy ion driver demonstration

heavy ton

heavy ion fuston accelerator research

High-mode

inertial confinement fusion, using D-T target pellets imploded by either laser or particle beams

fon cyclotron heating

ion cyclotron resonance frequency

inertial fusion energy

induction linac

Induction Linac Systems Experiment

flextble, medium-size tokamak destgned for easy access and rapid changing of the vacuum system and poloidal field system

International Thermonuclear Experimental Reactor 


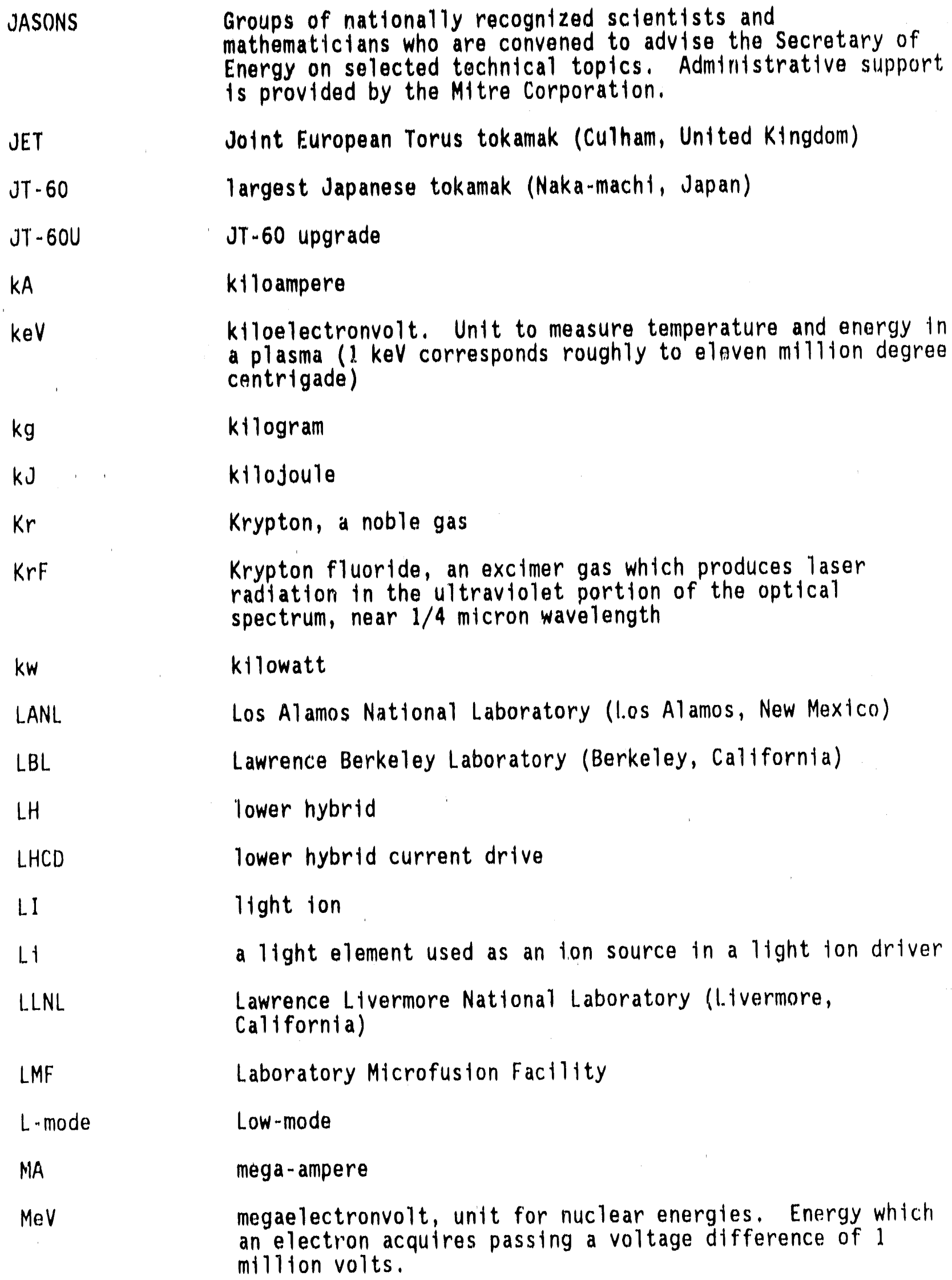

JET

JT -60

$J T-60 \mathrm{U}$

kA

keV

$\mathrm{kg}$

kJ

$\mathrm{kr}$

$\mathrm{KrF}$

kw

LANL

LBL

LH

LHCD

LI

LI

LLNL

LMF

L"mode

MA

$\mathrm{MeV}$

Joint European Torus tokamak (Culham, United Kingdom)

largest Japanese tokamak (Naka-macht, Japan)

JT. 60 upgrade

ktloampere

klloelectronvolt. Unit to measure temperature and energy in a plasma (1 keV corresponds roughly to elaven millition degree centrigade)

kllogram

kt lojoule

Krypton, a noble gas

Krypton fluoride, an excimer gas which produces laser radiation in the ultraviolet portion of the optical spectrum, near $1 / 4$ micron wavelength

kllowatt

Los Alamos National Laboratory (l.os Alamos, New Mexico)

Lawrence Berkeley Laboratory (Berkeley, California)

lower hybrid

lower hybrid current drive

light ion

a light element used as an fon source in a light fon driver Lawrence Livermore Nattonal Laboratory (l.tvermore, California)

Laboratory Microfuston Facflity

Low-mode

mega-ampere

megaelectronvolt, unit for nuclear energies. Energy which an electron acquires passing a voltage difference of 1 million volts. 
MFE

MHD

MIT

MJ

MTX

MW

$n$

NAE

NAS

NRC

NRL

$n r T$

OER

OFE

ORNL

PBX-M

PBFA II

PPPL

Q

RF

RFP

SABRE magnettc fuston energy

magnetohydrodynamtc

Massachusetts Institute of Technology (Boston, Massachusetts)

megajoule

Microwave. Tokamak Experiment (LLNL)

megawatt

number of particles in a unit volume of a fuston plasmia; particle density

National Academy of Engineering

National Academy of Sciences

National Research Counctl

Naval Research Laboratory (Washington, D.C.)

product of particle density $(n)$, time $(r)$ in which a fuston plasma is stably confined and the tempertaure ( $T$ ) of the particle ensemble (plasma)

Office of Energy Research, DOE

Office of Fusion Energy, DOE

Oak Ridge National Laboratory

Princeton Beta Experiment-Modifted, a tokamak for strongly shaped plasmas, (PPPL)

Particle Beam Fusion Accelerator (SNL)

Princeton Plasma Physics Laboratory (Princeton, New Jersey)

energy gatn, the ratio of the fustion power produced by a plasma to the amount of power that must be added to the plasma to sustain its temperature

radio frequency

reversed-field pinch, a magnetic confinement concept

Sandia Advanced Beam Research Experiment; an accelerator dedicated to developing lon beam generation, focusing and transport for inertial fusion appilications 


\begin{tabular}{|c|c|}
\hline SNL & $\begin{array}{l}\text { Sandia National Laboratory (Albuquerque, New Mexico, also } \\
\text { Livermore, California) }\end{array}$ \\
\hline$\tau$ & $\begin{array}{l}\text { time for which a fusion plasma holds its energy and is } \\
\text { stably confined }\end{array}$ \\
\hline $\mathrm{T}$ & $\begin{array}{l}\text { temperature (energy) of a particle distribution in a plasma; } \\
\text { measure of fusion power capability }\end{array}$ \\
\hline$T$ & $\begin{array}{l}\text { tesla; a unit of magnetic flux density, a measure of the } \\
\text { masnetic field strength }\end{array}$ \\
\hline$T-15$ & large superconducting tokamak (Kurchatov Institute, USSR) \\
\hline TEXTOR & $\begin{array}{l}\text { Torus Experiment for Technology Oriented Research (Julich, } \\
\text { Sermany) }\end{array}$ \\
\hline TFTR & Tokamak Fusion Test Reactor, largest U.S. tokamak (PPPL) \\
\hline TFTR (NBCD) & TrTR/neutral beam current drive \\
\hline TORE -S & $\begin{array}{l}\text { Tore-Supra; tokamak with superconducting toroidal field } \\
\text { coils (Cadarache/Fontenay-aux-Roses, France) }\end{array}$ \\
\hline UCLA & $\begin{array}{l}\text { University of California at Los Angeles (Los Angeles, } \\
\text { California) }\end{array}$ \\
\hline UR & University of Rochester (Rochester, New York) \\
\hline $2 T$ & reversed-field pinch machine (LANL) \\
\hline
\end{tabular}




\section{E.2 TECHNICAL TERMS}

alpha particle

Aurora

blanket

breakeven

burning plasma

Centurion-Hal ite

compact torus devices

current drive

deuterium

driver

excimer

fission nucleus of a hellum atom "He, relased in a D-T fusion reaction with an energy of 3.5 million $\mathrm{eV}$, which it gives up to the plasma

Krypton fluoride excimer gas laser (LANL)

region surrounding a fusion reactor core, within which fusion neutrons are slowed down and absorbed, heat is transferred to a primary coolant, and tritium is bred from lithium

for magnetic fusion: the point at which the energy from fusion reactions equals the energy lost from the plasma (e.g., through radiation processes). For inertial fusion: the point at which the energy from fusion reactions equals the energy supplied to the fuel

a plasma in which the fusion reactions supply a significant fraction of the energy needed to sustain the plasma

program of underground nuclear tests involving the inertial fusion energy program

a toroidal geometry for magnetic plasma containment in which no conductors or vacuum chamber walls pass through the hole in the torus

technique for making the toroidal plasma current using RF or neutral beam power, i.e., without use of an inductive transformer

a heavy isotope of hydrogen, ${ }^{2} \mathrm{H}$, which with tritium is a component of the first fusion fuel to be used; it occurs naturally in water

machine which provides the energy to an inertially confined fusion target in th? form of intense, high-power beams of laser light or particles

molecule existing only in an excited state; it cannot exist in thermal equilibrium with its environment

process by which a neutron strikes a nucleus and splits it into fragments; during the process of nuclear fission, several neutrons are emitted at high speed, and heat and radiation are released 
flux

fusion

gyroton

heavy ion

helium-3

High-mode

hohiraum

ignition

inertial fusion energy

ion the energy or number of particles per unit time and per unit area passing through a mathematical surface in space

process by which the nuclei of light elements combine, or fuse, to form heavier nuclet, releasing energy

device for producing microwave energy that uses a strong axial magnetic field in a cavity resonator to produce azimuthal bunching of an electron beam

ion of high mass (e.g., an electrically charged atom of an element from the middle to high end of the periodic table); in inertial confinement fusion, heavy ions are accelerated with linear (typically inducticil) accelerators

one of two isotopes of helium; the other is helium-four (see alpha particle)

enhanced confinement mode discovered by ASDEX in 1982; up to twice the confinement time of the Lmode

chamber for converting driver energy into radiant energy, usually for the purpose of driving an ICF implosion system

in magnetic confinement fusion, a plasma in which the fusion reactions supply enough energy to sustain the plasma without auxiliary heating

for inertial confinement fusion, a condition wherein the hot-spot fuel in the center of an implosion attains sufficient temperature and density so that its thermonuclear reactions not only heat the hot-spot further, but al so promote burning of the compressed pusher fuel

energy released from thermonuclear reactions in the fuel of an inertially confined target

an atom (or molecularly bound group of atoms) that has become electricaliy charged as a result of gaining or iosing one or more orbital electrons; a completely ionized atom is one stripped of all its electrons 
light ion

limiter

linac

low-activation materials

Low-mode

magnetic fusion energy

magnetohydrodynamics

neutron

Nike

Nova

ohmic heating

Omega an ion of low mass, typically an electrically charged atom or the bare atomic nucleus of an element near the light end of the periodic table; in inertial confinement fusion, light ions are typically accelerated across a small gap in a high-voltage short-pulse diode accelerator

device placed inside the plasma chamber to intercept particles at the edge of a plasma; by "scraping off" these particles from the plasma edge, the limiter defines the size of the plasma

linear accelerator; device for accelerating heavy ions (to drive inertial confinement fusion targets)

materials that, under neutron irradiation, do not generate intensely radioactive, long-lived

radioactive isotopes and do produce less afterheat following a reactor shutdown than high-activation materials

standard confinement mode observed in all tokamaks in which confinement time degrades as increasing amounts of heating power are input into the system

energy relcased by a thermonuclear reaction in the fuel of a magnetically confined plasma

the subject of electrically conducting fluids under the influence of electric and magnetic fields; its theory can be used to provide a good approximation to plasma behavior in many instances

an uncharged sub-atomic particle; neutrons released in a $D-T$ fusion reaction have an energy of $14.1 \mathrm{MeV}$, which is to be used for power generation and tritium breeding in fusion reactors

KrF excimer-gas laser, a major ICF driver facility, to be constructed at NRL

largest U.S. laser facility (LLNL); solid-state laser Nd:glass

the heating of the plasma resulting from its electrical resistance to the flow of current induced in the plasma

large Nd:glass-laser facility (University of Rochester) 
pe1let

plasma

poloidal

Precision Nova

reversed field pinch

stellarator

superconductor

supershot

target

tokamak fuel-containing assembly of an inertial confinement fusion target; energy irradiating the capsule is partially absorbed, driving a complex series of hydrodynamic processes, including the implosion of the capsule to small diameter and high density, and the heating of the center of the compressed fuel

an electrically neutral gas consisting of charged particles

referring to any $p l a n e$ of the torus that contains the central axis

the combined result of the improvements in beam energy, power balance, beam pointing, target diagncstics, and target fabrication capabilities required to execute the hoh 1raum physics and implosion experiments on Nova that are called for in the NAS final report

a closed magnetic confinement concept having toroidal and poloidal magnetic fields that are approximately equal in strength, and in which the direction of the toroidal field at the outside of the plasma is opposite from the direction at the plasma center

a toroidal configuration (pioneered in the US) in which plasma equilibrium and stability are achieved through externally imposed magnetic fields, without the current in the plasma required for tokamaks

a material that has no electrical resistance below a certain temperature; for the alloys used in superconducting coils for fusion research, niobium-tin and niobium-titanium, this temperature is <20 degrees Kelvin

operation of TFTR with peak density and strong central neutral beam heating

the entire structure placed where the inertial confined fusion driver beams are pointed in the experimental chamber; the target may consist of a simple flat disk of material, or may be a complex structure with many parts

toroidal magnetic confinement device in which the magnetic field lines of force close on themselves, with a large current flowing through the plasma; Russian acronym for "toroidal magnetic chamber" 
toroidal

broadly, in the shape of a torus (as in "toroidal configuration"); specifically, referring to the direction of rotation about the central axis of a torus

tritium

a heavy isotope of hydrogen, ${ }^{3} \mathrm{H}$, which with deuterium is a component of the first fusion fue 1 to be used; it is radioactive and must be produced using neutrons 

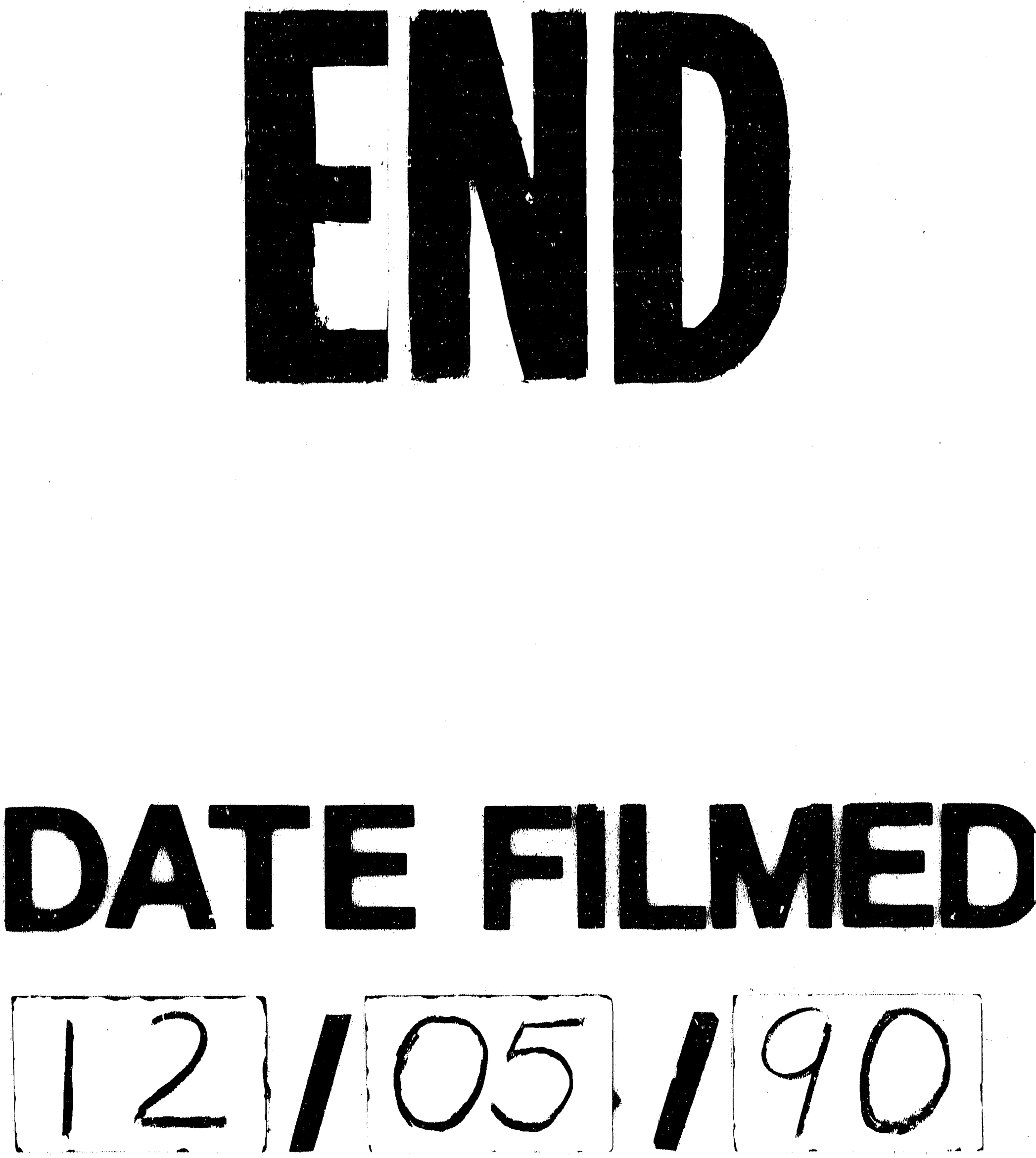
